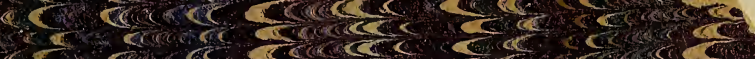

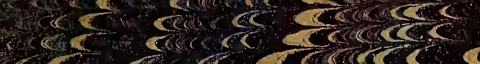

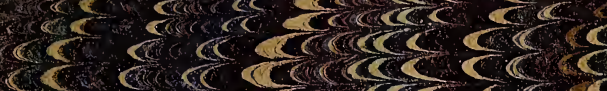

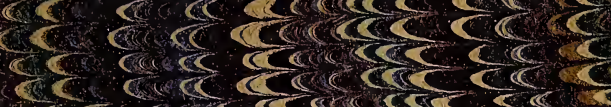

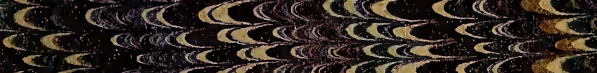

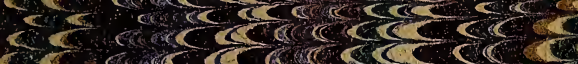

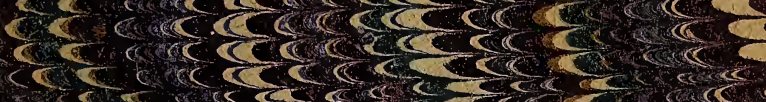

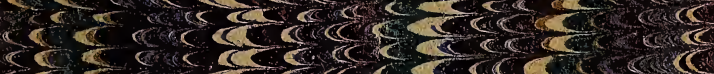

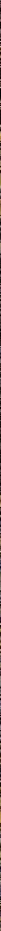



ang. 





\section{.24 \\ ia 52}

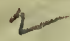

\section{2}

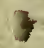

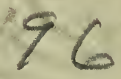

\section{HYMNAL}

SENECA INDIAN LANGUAGE

\section{SANBORN}

PDTFT:AT OBE

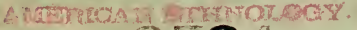

$$
\therefore=3
$$

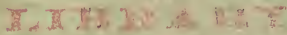

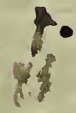





\section{HYMNAL haples, N.Y. \\ IN - THE \\ 95 \\ SENEGA LANGUAGE \\ ALSO \\ TEN PSALMS OF DAVID}

TOGETHER WITH

A CHOICE COLLECTION OF ENGLISH HYMNS WITH TUNES AND AN INDEX •

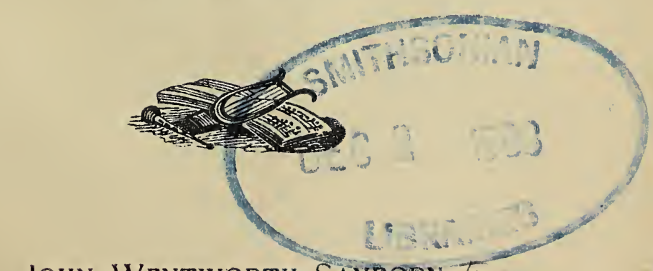

EJOHN WENTWORTH SANBORN tra

O-YO-GA-WEH, Clear Slcy

1892 


\section{pM2296}
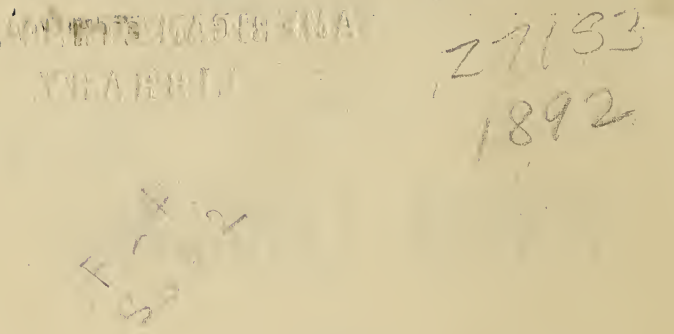

\section{NOTICE.}

Oh ka dooh ah dah geh gwa heh ga ă năgo nyoh neh no gwah no ya do nyoh nă gă gaă năo nyoh.

Translatron: I have put under each hymn the name of the person who wrote it. 


\section{PREFACE.}

In this book are only those hymus which the Indians will use. I am indebted to Rev. Joseph Turkey, and other Indians, for helping me translate Psalms and hymns now first printed in this language. I have also compiled some hymus translated years ago by the late Rev. Asher Wright. I have had to set all the type of this book with my own hands. I printed it also on my own press.

The translating, type-setting, and printing have taken nearly all my spare time for the past 14 years. The work has been slow because I never learned the printer's art. except as I have acquired some things in making this book. Printers will see imperfections in it, but I determined that the Indians should have a hymn-book, and here it is.

It is right to express here my thanks to James Conners' Sons, 'Iype-founders, of New York, for courtesies in making this Indian type for me. Gratitude is also due to the authors and owners of the English hymns and tunes, for allowing me to use their choice, inspiring pieces. I trust God's blessing will go with this modest book.

JohN WentworTh SANBORN. 


\section{A GULDE TO THE PRONUNCLATION.}

Capitals

A.

$\check{\Lambda}$

A. $\cdots \cdots \cdots \cdots$

$\underline{\mathrm{A}} \ldots \ldots \ldots \ldots \ldots$

F..........

$\breve{\mathrm{E}} \ldots \ldots \ldots$ e

I
Small Letters

English Sound

o.................

o.................... ong (long o)

$\mathrm{U} \ldots \ldots \ldots \ldots . . . \ldots \ldots$ in bush.

v......u in $u s$.

v........ oun in young.

$\mathrm{Ch} \ldots \ldots \ldots \ldots$ ch $\ldots \ldots \ldots$. cb in chime.

$\mathrm{H} \ldots \ldots \ldots \ldots \mathrm{h} \ldots \ldots \ldots \mathrm{b}$ in $b e$.

$\# \ldots \ldots \ldots \ldots$ in $o b$.

No letters are silent. A hyphen mark - under a letter gives it a nasal sound. ai should be sounded like $i$ in mine. $\quad i u$ like $u$ in pure. $s$ never sounds like $z$. $h$ has its full sound after $t$ and $s$. $\quad e i$ sounds like $a$ in day.

The large spaces divide words; the small ones divide s llables. The letters $b, f, l, m, p, q$, $r, v, x$, and $z$, are not found in the Seneca. 


\section{SENECA HYMNS.}

\section{WORSHIP.}

C. M.

Exultant praise to the Redeemer.

$\mathrm{O}$ wăh nyah eh shoh teh gwă not Neh da eă săo nyo ok;

Heh noh deaăh săoh Na wen ni'yuh, Kuh heh nioh da ni dăoh.

2 Tis sgioh nya nih Sgwă ni yuh kuh Da gye no wos no năhh,

Dạa ga do gwat neh yoăan jah geh, Heh nih sah să no noôh.

3 Christ huh să noh yoh nes núh dyot! Kuh noh săt ă yoh nek, A ya gaoh es hah nă yo ok, Neh goi wa neh a goh.

4 A ja go da dwă ni yoh het, Yeh ho doh wáet găh geh;

Neh nat gwăh sah ăhs ga noa eh;

Ga gwe goh 'deh wi yo. 


\section{Worshiping the Lamb.}

Ga oh' da swet', do găa ${ }^{\prime}$, wa doh, Do gắ, kuh, ae dwa' dă no dặh Neh na dío yah̆ ge o nah̆; neh, Naeh, Na wěn ni yuh̆ ha nọ ehs.

2 Do gă dă yó gwa deh' să dook $\mathrm{He}^{\prime}$ o weh nit ha dyăn' dah gwah Na yah̆ da ğwěn ni yŭh, neh,, naeh, Neh neh' huh dyuh' ni goi' yus deeh.

3 Neh naeh, we suh' wăh nyah̆' es yoh̆' Ni ýuăh nợ' sa gee, neh neh huh' Deo wăăh' săgo nyoh Na' wěn ni. yuh, Neh huh', naeh, dya wăn det gă deh. 4 Skat dih', sho, năh iih hae' dwa dōh, Des she dwăăh săo nyooh neh Je sus; Neh, săhăh, dyuh ni goi yus deeh

Hă yui' wa da dyı̌ĕ deh o dohk.

Isaac Watts.
$: 3$ (METRE CHANGED.)
C. M.

\section{Invocation of the Trinity.}

Ga oh' da set', Se go' wa năhh,

Neh Syah́ da gwěn ni yuh;

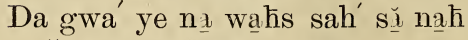

Á ya gwa di no thes.

2 O Je sus, Nis Sgwa' wěn ni yuh, Sa' dyah da gwa ih sih,

Des he' ya dó gwat no khi' swạệs

Is, kuh, she eh' datis. 
3 Neh Nis seh has', des yo' wa nğh Neh o gwa' ye na' wı

Neh na yo gwa' yah, da' ge hah

Gá yă năn' dah ó ak.

4 Ga jih', 'Sa yah̄' da dó gạh dih, Skă noh sheh ni' goă y⿳亠丷,

Neh he ni yá oh e sut, neh

Nis, oh si' wa' ni yát.

5 Do ğs seȟ has' des yú wa nệh;

Is dènt chắ, nch dó ok,

Neh he' ni gá wén yah sa ge,

Sá noh, naeh, ă sa dih.

\section{1 \\ Charles Wesley. \\ L. M.}

General invitation to praise God.

Ga gwe' goh neh dwa na g' nyoh

Níeh dah ge' g’wa nạh găọo ya dih,

Neh a' she dwai' wa yă ni aak

Je sus, sho gwa' yah dih' sah oh.

2 Ho dá ni dă os yo wa năh:

Nă yui wa da dyı̌ěh' go dă oh,

Do găs' no wại wa gwěn ni ' yus;

Ye í wă a a $\bar{h}^{\prime}$, kuh, na' wă nạh.

Isaac Watts.

5

L. M.

Invitation to worship.

Iis naa hah' de jo ăn joh dăhh' shoh Neh 'yu ăn' jah gĕh, jō̌' e shah $\mathrm{He}^{\prime}$ a gat Na wěn ni yuh, neh Das' he swěn noh' do ni' a dyiěh. 
2 Neh Ná o hwạh Na' wĕn ni' yuh Sho gwa' wis neh dyo heh' goh, kuh No gyó is hăăt: há o hwạ $\bar{h}^{\prime}$, kuh Ho i ch dves hăh he dyo heh. $3 \mathrm{Hoh}^{\prime}$ swa yoh' hot ă o ga' ănt, Des she' swa no nyo neh; neh, kuh, Neh ă swá dye ăh' dah gọ ok Neh des' she swa ăăh' să og nyo okk.

4 Shoó gwăn dăa ăs $\mathrm{Na}^{\prime}$ wěn ni' yuh: Ho da ni dă os yo wa năh;

Neh, kuh, neh goi' wa ye is doh, - A ye găh he ni thai wa yeih.

William Kethe.

(i)

\section{Blessing on worshipers.}

Hes ga gont neћ Sgwa wěn ni' yuh, Da gwah dăh swi'yos dặh,

Să noh ă yo gwaé nos da neh Na gwái wa yắni ak.

2 Hah ni, da gyoh nă yo gyo het; Je sus hoh să no gọ

Å dyo givah ni goă diă wă ọk, O gwát wai shăh je nıِahh.

3 A gwa ye nạ ah kuh ne swă noh,

O gwă nyah sa gôh jih,

Á ya gwa sı̌ no nyah jih kuh, Tăh' to sa yo gwah do. 


\section{WORSHIP.}

4 Neh nă yo gwă nyăh sa g’we gọ, Da gwa dăh swi yús dăh

Wa do dyak kuh no noh gwă oh,

N'eh sah haћ shah ăo dih.

\section{Joseph Hart.}

y

Thanksgiving for infinite love.

$\check{\partial}, 6$.

Is, no' hah̆' shăh shoh

Na' wěn ni yuh, neh̆

Neh ti' ga g'we goh

De swa di o gwat

He' nioh' să nọ neă g'wat

Neh Cha gạo he dus;

Neh kuh, ne' ga g'we goh

Da o' noh don dyiěh.

2 Neh $\mathrm{Na}^{\prime}$ wèn $\mathrm{ni}^{\prime}$ yuh

Cho, gwěn' noh do nih

Nǐk' huh yoăn', jă gěh ;

Neh kuh, naah', haas deh

Neh ắ cha gá o het;

Nęh na, ó gwe, dah

Ă ,yon' dă no, dah gwaak

Ho yah' da n'éa gwat.

3 Neh Na' wěn ni' yuh

Ho' yah' da néă g,wat;

Neh' neh ga gwe' goh

De dwaăh , să o nyooh

$\mathrm{He}^{\prime}$ ni oh' să noh dă;

Nó da' ni dăes' hăh,

Sho giva' da dih nŭh' it,

() gwai' wa' neha goh. 


\section{She dwa' dă no thvs'}

Neh Cha găó he dvs,

Neh, kuh, něs' he dyoh

Neh de gạăh' săă oh;

Neh na dío yah̆ geo nōh

Deó wạăh' săa o nyoh;

Haeh gwah', dih, năh ih, neh

Dạ́' she dwăăhh' săgó nyook.

Charles wesley.

\section{Dismission Hyme.}

For the fullness of peace and joy.

O Swěn ni' yuh, don da' g'wat gah,

Iis da gwa' dăh swi yus dăh:

Da' gwa nont' he ni sek' wi yu;

Ne swă năh o gwá ye nạ;

Da gwa' noh heh' dăh nạoh' e sat;

Da' je nạh neh o gwạa $\underline{a}^{\prime}$ nah

No năh' hă yá gwa yoh chih' dyoh,

Dyut gont' dă gwăăh' săo nyó ok

Dyut gont' dă gwạăh' sịo nyo ók:

Á ya gwih', Hal le lu yah,

$$
\text { A men, Hal le lu yah, }
$$

Å ya gwih', Hal le lu yah,

A men, Hal le lu yah, ne Ha wĕn ni' yuh.

Hal le lu yah dya' wăh oh,

Hal le lu yah deh' o dohk,

Hal le lu yah ha' yu i' wa da dyiěh', A men: Hal le lu yah, A men, A men, A men. 
Joy of public worship.

O Swěn ni' yuh, da gwat' gat huh,

Iis, săhh' ăh, sgwa yah' da ge' hăh;

Sat gat huh ha di' yah da deh

No gweh, Iis ji só gweh dah̆' shoh.

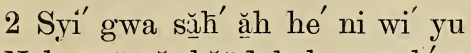

Neh swă nǐs hăăd heh sa noh' sot,

Na' eh no dă nĭs hă gah deh

No ga' dăn det' gă' don dy̌̌̌̆h' sek.

3 Neh sah' gwah a' gă da' dă gwv's

A geh' hoa no nohk sa noh' sot,

Naeh neh da' ya gwa' di aak neh

$\mathrm{Nah}^{\prime}$ de ya goí wa ge no gweh.

4 De yo gwa deh' hat hehs' dah goh

Ha yah̆' da deh $\mathrm{Na}^{\prime}$ wěn ni' yuh;

Neh kuh, dwah has' des hă' dah goh

Dya weh' dah goh ha yah'da deh.

5 Neh kuh né a hwăh sho gwa' wii

Neh o gwah' ni goi yus dah goh;

O Iis, ne Syah' da g'wěn ni yuh,

Go dạ ôh no sai' wa yă nih.

10

Isaac Watts.

C. M.

\section{Renewed consecration.}

He' sga gont', a gat' wa is' hăh,

De juh' hat heh năn' deh.

Sa yo, yohis' ne se gá, ah gěh

Sah' ni goi yus dah' goh. 
$2 \mathrm{He}^{\prime}$ sga gont', a gât wa is' hăh, Neh do sá e snó nyooh

Neh Thă noh' dọ ạh găó yah gěh, Kuh neh yo ăn' jah̆ gĕh.

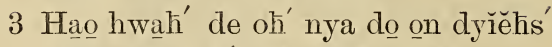
Ne gyah̆ daȟ' gěh; neh, kuh, Neh găh noh's sah ă gev' nyieh dvk Neh deơ ăh să o nyoh.

4 Neh̆ nih̆ a gi wa neñ ${ }^{\prime}$ ak shăh Ho' noh $\bar{h}^{\prime}$ kwăh syu' nyah thah; Ah' soh dih, hoă' ni goắ ni yoh,

Neh kuh, na' gi dö ăs.

5 Swèn ni' yuh, Is Se' go wa năh', Da geñ̆, has dis' yo nih, Nó sa goó yath da giwen ni yus He' ni sğă niss ha wii.

Isaac Watts.

11 C. M.

Preparation for public worship.

Se deh' ji ah ăh sat' hon dek,

O Sya $h^{\prime}$ da g'wĕn ni, yuh, Na ga' dì ná yăn dah, g*wah shăh, Heh tgặh a ge gon' dvk.

20 non' da gă yat' gwa, hes hehs Neh Christ, Cha gado' he dvs,

Cha go' doe syoh gwa H.a gat Na' wěn ni yuţ.

3 Iis sa g'we' nyo na' she yo het Ne goi wa ne"s' a goh; 
Neh set, gwăhh' sah̆ ă yon' dyeăh' dvk Á jon' dyah da' go e⿳亠口冋.

4 Neh dih', sho, he' di sa' noh sot Neh huh' hă sgih' dyon dah̄k;

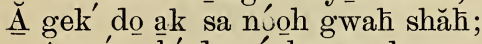
A gó yah̄' da núoh gwaak.

$5 \mathrm{O}$ da se' nyet neh Sat wais' hăh, Ha' wak haah, a' gye is $\mathrm{He}^{\prime}$ ni sai wih' sah̆ oh, neh kuh, Nơ sa gọ́ ya nă $\underline{a}^{\prime}$ a

Isaac Watts.

12

Evening meditations.

L. M.

Neh̆ huh' ni yóo weh' he ga wihs

$A h^{\prime}$ soh Na yah'da gwěn ni'yuh

Ha găánĭs hă gạis'da nía dyiếs;

Oh găăs'ah ah soh tga ye ih.

2 We suht', noôh, noh ga'dye sat' sho:

Dus găh', kuh, nǐs gehs', hăăs'sna g'ek'

Des hái wah̆'să gwơs neh nậh' gạa

Ha gyaȟ'dani yăăs nak' hăa do.

3 Oh ga'dyvs hăăh ăáwa gi' dał;

Neh noọ yah ge o nō̄'shăh shoh

Dăoó găh nya dọk, ni wah'son dis,

Dạa nạn deh̆'să doōh nak'nạk dah̆.

4 Neh huh' nă yáwăh năss gi' eh;

Q eh'da go ăs gá dyus hăăh̆,

Ă e'yus haek, neh ă a gyet,

Ăs ga'doh het neh gặo yah gĕh.

Isaac Watts. 


\section{GOD.}

PROVIDENCE.

13

The pilgrim's Guide.

$8,7,4$.

Da gọ want has, O Swěn ni'yuh

A gyah'dah doh ${ }^{\prime} \underline{o}$ on dyiěhs:

Deh geh'has deh, Tis seh'has'deh:

Iih da gye'nạah šs oh'dah;

Gak'wa no'oh',

Dak nont' ă wá gah dah'jih.

$2 \mathrm{O}$ seh ho' do guh o doo sot, Neh huh' tga noh' gwah syu' nyoh;

Oh ha' ot ă wak ha wih'sek

Dya wăhh oh, he gat'hái nehs,

O seh'has deh,

Iis sho, dak'ni goó ni aak.

3 No năh găhh hak'ah ne Jor dan

Dăk daht, O sah gwih' noh'dyont;

Skă no noh jih da gyatíd da wă ă ănt,

Sgăgh'hạn dih, ne Ke nyặn gwa,

Gạa ă nạh shōh,

Dyut gont ạ ga dặ no' thaak.

William Willams. 
14

S. M.

Infinite compassion.

O a gat' wa is'hăh, De eh'săh,săo nyó ok

Na'wěn ni' yuh, neh dyu'i wah

No dá ni dă ós hăh.

2 O gwai' wa neh'ak shăh

Hoh'ni goăa et gănt hah',

Neh gwa', sho, he' nio da'ni dăaoh,

Neh chọ́ gwặn dạăás dah gwah.

3 No năh' ă shơ g’wa yěnt', Än dyu' no'ôh, naeh heh

Ni yo gwai' wa' neh ak'syuh dạ ; Tặh ăh deah' nya gaas deh'́

4 Neh he ni ah'haas deћ̆,

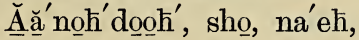

A'gi wah'dohht neh o gĭs'swạăs;

Neh hoh' ni goăáni yoh!

50 i'wa ne'ă gwat, Na yah̆'da gwěn ni yuh,

Neh he' ni sha' go no oh g'wah

Neh dya goi' wa yeis' doh.

6 Neh de sga' yắon daah, He'ni ye no' oh gwah

Nógweh, ne guk saȟ'da yăn dô ${ }^{\prime}$,

He' ni shó gwa nóoh gwah.

Isaac Watts. 


\section{CHRIST,}

\section{SUFFERING AND DEATH.}

\section{5}

C. M.

Godly sorrow at the cross.

Hot gwăh sai' oh Cho gyoh'hee dvs, Do gặs' ha wee yos'oh;

O giwe nyos oh' hà gíe yaȟ'seeh Niih', héni dwa ga dohk.

2 Neh' găah dyoí wah ha gyăh hih oh Hos yo' dyă

Wa da ni dă ós yo wa năh!

Do găs', naeh, hak núoh'gwah.

3 Oi wa yăăs doh' ăăh̆, ne găăh gwaah, Son da gooó waa' dah set,

No năh' Christ sha ă eeh, dyoi'wah

No gwai' wa' neh aak shăh.

4 He ni wa' gat goh'sa neh' wăoh, A gi'wa neh'a goh,

Deh oi wá yăs doh găo yah gěh,

Haa' gat goh'sa gah' dăh.

5 Sah gwa neh no gah'să gooh jih', Niih, aa' gat goh" sah set;

No nŭh' he de a' yah son dvk',

Neh huh' aa' gat gaat hoh. 
Glorying in the cross.

Neh huh' no năh' oh gat' gat hò

$\mathrm{Ne}$ de ga yah sont, he'o weh'

Ha wee yos'oh Cho gyoh hee dis,

Neh' sho niih noh̄k hni gó et gặht.

2 Deh thah' gwĭs dặ sho, oh waa dōh,

Ne' gyah daa ahs' găăăs, yoăn jah gěh;

Neh sho neh', ohk hni go et gặht

Neh, he no gi dăs the ht go gweh.

3 Syah da gwěn ni yờh', da gáis dăh,

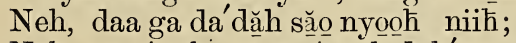

Neh gwaa', sho, ă ges'nyeh dah' g'waak,

He'nis sho gwạn dạ oh Je sus.

4 Sat gaa thò has oh' dah ges hoh,

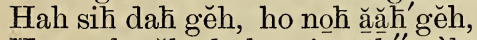

Has wah gěh, kuh; ni ga ăă $\bar{h}^{\prime \prime}$ sòh jih

O dạa ă ăht nió yah da'wặh oh.

Isaac Watts.

18

7.

Christmas Hymn.

TUNE: I am trusting, Lord, in Thee.

Na di'o yah' ge o noh

Yoăn'jah gěh ho di' yó nộ,

Neh ho di'wa yặn da dyeeh,

Si'gwa oh' ni goí yus deh.

\section{CHORUS;}

Hă nạ dọ, Neh nă gắ wă nĭs hă deh wa ă na găăt

Ne Je sus Christ, ă cha gaonh het' nó gweh, neh găo ya geh. 
SUFFERING AND DEATH.

2 Neh nó gweh hó wạn di'găh

Si' gwa găn dyuh' go wa năh:

Ho di' yah̄' da do' găh dih:

Gạă nạ seeh ho non' dạa not.

CHORUS.

3 Ho nă , Să noh' ă swah' dyok;

Neh săh̆ăh oh gwai' wae wăh

Neh skă noh' ti gai wi yooh

Heh swa yah̆'da deh jo gweh.

cHORUS.

Author unknown.

18

L. M.

Dying, rising, reigning.

Wa ă ê ne Cha gaóhe dvs!

Ga oh', da swet', swai' wi yus'doh,

De swa' gah sắnĭs hăh'syó , kuh,

Neh săh ăh, nis oh she swăe yahs'.

2 Nǐk'huh ga yăh no nóoh gwah'shăh,

Neh kuh, noh' ni go à et gănt;

O ăăh'dah goh oi o wa năh ';

Deh o' gwe nyo na' yont yuo wih!

3 Neh neh Ho yah̆'da ne'ă gwat

Sha ga'we e' yah seeh nó g'weh;

He ah'sạ dok yo ăn' jah gěh;

Neh gwa, naeh, neh sho doh'he doh.

4 Neh neh shot gah' wăh heah'sa dok;

G.̃. o yah ge.̃ he'sha we' no;

Neh na di'o ya $h^{\prime}$ ge o noh

He shó wa yah' theeh Hoh ni' neh. 
5 Swắni hăa des she'swovs dă nih, Swai'wi yus doh, swat, yuo wih' gwa, He ni eh tgăh neh găoo yah gĕh Ne'wah he Cha go wèn ni'yuh.

6 Neh sa swa' dă no' dah goó ok, Hoȟ'shă nyooh' neh gah has'des hăh Na nuss'he ó noh gěh, neh kuh, He ni gah nya gaas deh găa yaht.

Isaac Watts, alt. by J. Wesley.

\section{THE HOLY SPIRIT.}

\section{9}

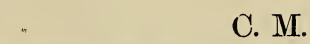

His quickening power.

Ga oh', da', set', Sat wais'hi yu,

Don' da' gwah'ni goi yus,

Sa sa de'gah̦t ă swah dai'yaht

No gwèn'yąh sah⿱' gěh shoh.

2 I dwe', da sha gwaăhh'săónyo ok

Na yah̆' dā, gwěn ni', yuh;

Neh, we suth', gwen' yah sá no,

Tăhh' deh ja gwa yặh'hehs.

3 Syah'da gwěn ni' yuh, dyut gont', găh, A yagiwă é yoh sek?

Dya gwa'dohk thah niił, gwa núoh gwah, We suh' niis, sgwa núoh gwah.

$4 \mathrm{Gaoh}^{\prime}$ da'set', Sat wais'hi yu, Je ăh̆t' seh has' des hăh,

Neh ósa yo gwăh' ni' goo nyaht Je sus ho n'joh gwah'shăh. 


\section{THE SINNER.}

PROVISIONS OF THE GOSPEL.

29

The joyful sound.

Ja go yah̆ da' gwat hah no gweh,

Do găas gai'wa gah'oh;

Sga yăhh'săoh'gwah de yu'dyah khoh

Neh gah'ni go ăă'gěh.

2 Goda'deh sa' dō waet' gặh gěh

Yeh di goo ă et găhs:

Dus găah he ya'go da'dih dyoh

Na nis'he ó nạ gě̀h.

3 O năh dih don'de dwat' ges guh

Neh dó sa yó g'wv's nyeeh;

O'se dwa găah neh găọ yah gěh,

Noh ni gó i yus deeh.

4 Ja go' yah da' gwat hah no gweh,

Jo wă nă is' da noh,

Tho nạn deh' has dôh neeh tgăah' shoh,

Neh des ho wăăh's săo nyoh.

Isaac Watts.

21

C. M.

The gospel feast.

Swa daoh' di yòs nee' ga gwe gọh, .

Swa doh'hoăh' ne jogweh', 'Tga wăă noot' ne gai' wi yòs dvk,

Ga gwe goh' dyo g'wăă noos. 
PROVISIONS OF THE GOSPEL.

2 Swat hwais'hăh, ăn daћ, o nyòs'hehs; Găă hah', sho, yo heh'goh;

Yoăn'jah gĕh' a wih'sa gon'dyiehs,

Naa'yò ni'goi'yòs dvk.

3 Năăh̆h" gak hwa'eeh, nă jaoh'hees dvk',

Găe yos' ne sat hwais'hăh;

Soh heȟ'oo weh', naeh, năh'seek dooh,

De wăn doh thaah'si eeh.

4 Iis' ne dyòt gont', swaah'dat hăh'sehs, Gah hăs'thah swă ee yos;

O doos hòòt' năă, năn ji'soh heet, Neeñ' sat haȟ'da noo wănt.

$5 \mathrm{O}$ doh'hon doh ne găhh' něk huh, Ja go'yah da'gwat hah;

Tăhh ăh deh̆ os'thooh deh̆ o găăh,

Nă sa'yah da'geh hah.

Isaac Watts.

WARNING AND LNVITING.

28

$6,8$.

Come to Jesus, just now.

Ga oh' daa seh, ga oh' daa seh

Ha yah'da deh Je sus;

Je sus ha yah'da deh

Ga oh' daa seh ne'wah.

2 Neeh ă syo'heht, neeh ă syo'heht;

Neeh ă syo'heht' o năh;

Ne wah̆' wă nis'hă de⿳亠̆

Neeh ă syó'heht' ne'wah. 


\section{WARNING AND INVITING.}

3 Ho deh'sah oh, ho deh'sah oh,

Ho deh'sah ô o năh';

Ne'wah ho deh'sah oh;

Ho deh'sah oh̄' o năh'.

4 Neeh he jah'dăht, neeh he jah'dăht,

Neeh he jah'dăht o năh';

Ne'wah neeh̆' he jah'dăht;

Neeh he jah̆'dăht' o năh.

5 Neeh he se guh, neeh he se guh,

Neeh he se guh o năh

He ji'wa g'wěn ni'yus

Neeh he se guh o năh.

6 Hal le lu yah, Hal le lu yah,

Hal le lu yah, A men!

A men, Halle lu yah!

Halle lu yah, A men!

English.

28

$$
\text { Inviation hymn. } 8,7,4 \text {. }
$$

Ga oh' da'swet', iis, ne jo'gweh,

Iis, neh swai'wa neh'a goh;

Ga oh' da swet', he'ni ga'yăh;

No'da'ni dạos hăh Je sus;

Ho deh'sah oh

Neh ă ji swa'yah da gwat.

2 Să noh' dăh saĥ'ni gọa ge'ak

He nio da'ni dăcs yuh dă

Ho gwe'nyo, sǔh ${ }^{\prime} \underline{h}$, nă ${ }^{\prime}$ yoh het,

Neh ni sah'ni goi yu'ak

Găo yah gèh,

Heoweh' dyuh̆'ni goi ${ }^{\prime}$ yus deeh. 


\section{WARNING AND INVITING.}

3 Ga gwe'goh, dih, neh huh' hae'dweh', Heh dyuh ni go'i yus deeh;

Neh na'yogwa'dăh swi'yuh heћt,

Nae'dwa dă no'dăhh, nae'dwih',

Halle lu yah,

Je sus cho gwa'yah da gwăh.

James Hart.

21

$$
8,7,4 .
$$

Come, and welcome.

Ho weh heh dyontgěn'nĭs at'hah

Neh da wa'dwă na'gah dặh :

Neh̆ no'hah'shăh Na'wĕn ni yuh

Des ho gwai'wa gwaih'da nih

Neh gai'wi yuhs;

Dwai'wa ye naagh' neh dyo'gweh.

2 Neh no gwa'yah dah'doh ó'nō Ha yaȟ'da deh̆ ne Je sus;

Det cho'gwă nos dạ di'dwah get;

Neh wi yu sho gwa'da di:

Neh gai'wi yuhs

Dwai'wa ye naahh' neh dyo'gweh.

$3 \mathrm{O}$ ho da'ni dăosos'yo wa nạh

Neh Je sus, cho gyoh'he doh,

Neh sho gwa'dyah don'dah gwa nih

$A^{\prime}$ gă a a $k^{\prime}$ deh ơ gơ

She dwa'núoh gwaak:

Dyut gont' she dwa'yah dăăh'sek.

Thomas Haweis 
Turn ye, for why will ye die!

A naeh' ă'swă'eh, O swai'wa neh'a goh?

Dus găoh' ni eȟs Je sus, no'sa she joh het:

We suh̄', naeh she swăn'dăăs, de she'swa ga'neh Ho deh'sah oh nó sa she'swa yah̆'da guh.

2 O năh', kuh, No twais'hi yu she swă'nak heh, Neh dáe swah ho'ón di h neh swăn nyah'sah gěh Neh, dih, theh̆'wa dye săh, hah soh' she swa'noh Neh na'wa dyiuȟ'dat neh swăn nyah'sa gǐs shōh.

3 O neh theh o'gwe nyooh' nae'swăn ni'nạn dok Heh we suh, oh'dyont, he ni swa'yah da wăs; Neh dae'swah sai'yăh no'sa swa'dat he wat, Nae'swa da'dat gah Je sus has oh'da⿳亠 gěh.

4 Onăh' ă dyoh'e sat neh gă’o yah gěh; Ănt gai'wa nạ găh noȟ'ni go'i yus deeh, A wann'do ok, "No di'yah dah'dōhónoh,

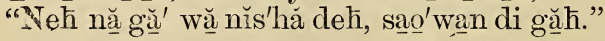

Josiah Hopkins.

\section{6}

\section{S. M.}

\section{The second death.}

Gạo'g'wa' noo', ă ye'găh

Neh yon dois hăn'dah gwał,

Neh de yes'găeh neh gut wais'hăh

Neh goi'wa neȟ'a goh?

2 Neȟ ti yuh'ni goohk'dvk

Nă yon dă nah⿱'ge ăd

Ti yóa ja g'we'goh, do gaht'

Heh gắo ya de'nyoli. 
WARNING AND INVITING.

3 Neh neh yo ăn'ja deh De'wăn doh ta'ya gạoh Neh ă'yuh'ni go'i yus'duk Neh gut wais'hăh no'gweh.

4 Neh neh do'găs o weh Ya go'heh, tặhăh, naeh,

Deh neh huh', shọ, na'ya go hek Nǐk huh yoăn'jă gěh.

5 Haeh'gwah', kuh, ne găe yaht, Tăh̆ăh neh huh' deh neh Na'ya gó'ya găa'ak, neh, kuh, Na'yon dọ'is'sweh dặh.

6 Nèh gwa', do gặs' tga yặh Neh' neh yoh heћ'o weh, Ga gaas'deh, tặh ăh deћ'o dohk, O i'wa da dyiěh'gĕh.

7 Tga yăh, kuh, neh gặe yaht, Oh'dyont, o ji'wa gặh, Deh o'gwe nyo a'yont yuo wih He nio'núok des yuh'dăh.

James Montgomery.

29

S. H. M.

Quench not the Spirit.

Ho di'ăh do'on dyiěh

O gwa dăoh'shoh găáaah;

Deh̆ gai'wa'gas deh he'dyo heh

Nik huh yo ănn'jah gẹh;

Neh gai'o nih deh os'haeh'gweeh

Nǐk huh a'yo gwa dōis'hăn'dahk. 
2 Si gwa nah̆ wann'dih gěh Dyon da'deh sa'doh gwah, Neh ho'o weh dyo ăn'ja deh Neh oh ni goi'yus deeh: I dwe', neh huh' sha di'na geh Yoh heh'o weh sho non'doh oh.

3 Ta gai'wis heht, o năh

Tih, kuh, ă dwat gat huh

Cha go'des ah'seeh ne Je sus

Hao hwah' ha o'gweh dah;

Iih, kuh, do gạa àn ji'dwa doh

Neh nik huh o khi'yah don dyooh.

4. Hoo'weh ă je'thi găah

Nik huh e'thi núoh grwak

Tah dae'dwa dek'hah sih, wăn doh,

Hă yò i'wa da dyiěh;

Do gă dă she dwặh'săgányo ok

Ne Je sus, Cho gyoh'he da'noh.

Mrs. Ann B. Hyde.

28

7.

Sinners turn, why will ye die!

Do sa'swặh ni'goăn de nih,

Tis neh swai'wa neh'a goh:

A'n?h oh'd d.h' dyờ'i wah,

Heh swa ga'yăs năg'swă eeh'?

Neh She swaち'nih, tgăo yah gěh,

Shes'swáon doạh, ha dọh, "A naeh'

$\mathrm{He}^{\prime}$ go waah heh swe'noon dyiěh'

He de dyu'doh ho ga nyast'? 
WARNING AND INVITING.

2 Don'de swat'chih gwat huh, dih;

Iis swa yah'dah dōh'oon dyiěhs'

Neh She swa'wěn ni yuh Christ

She'swáon dọah', ha doh, "A naeh',

He'go waah' heh swe'noon dyiěh'

He de dyu'doh ho ga nyaht?

A'nah oh'dăh' gai'o niȟ'

Iis swa nó'eehs nă swă eeh' ?"

Charles Wesley.

28

$$
9,6,4
$$

Almost persuaded.

O năh tho hah ă skni gọ da goh, Neh na gi wa g̛̣ă ni yòs o năh,

Christ a e goh' să ăh ăh

Neh gwa'neh sga do wih;

Neh neh noh găăh ${ }^{\prime}$ ya ek

Hă sgón nook naeh.

2 O năh tho hah̄' ă skni gọ da goh, Nă gặh' wă nis'hă deh dạnt ga'get,

Je sus tha gi'nos kuh,

Å ga'dă na yăă dih,

O a gyah'dah doh oh

Ah sga' dạ dih

30 năh tho hah ă sga yạ twă gwăok,

O,năh tho hah' oh́ dyont ă wo khăt,

Tho hah' săh ăh o năh, A wo gah doō' gwah ho Oh săt' thah wa dok'dăh,

Nă wo gah'dooh. 


\section{REPENTANCE.}

BB

The Son of God in tears.

Neh, waeh, neh Cha ga'o he dus

De sha'guh sănt'hwah seeh

Ne goi'wa neh'a goh no g'weh,

Neh go yah'dah doh'oh.

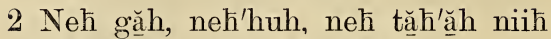
Tah don'de dwa'gah săiht?

Oi'wa néă g'wat na'gă ak niih

Deho'gwah'ni go'nyah thah.

3 We suh' wao di'wa nă'ă guh

Na dío yah'ge o noh,

No năh, sạo'want gat huh' Je sus,

Deo'gah sắnís hăh̆'syoh.

4 Sai'wa néă guth, haeh'g'wah, năh' iis, O a gat'wa is'hăh;

Neh, săh̆ăh, niiis de yv's'àa niih

Neh Cha ga'o he dus.

5 Neh gai'o nih neh has dăăh'g'waȟk, A $^{\prime}$ yo gwa'nok do thus

Neh do'se dwăh'ni goăn de nih

Ho gwai'wa'neh a goh.

6 Neh săh̆' gădo 'yah̆ gĕh ta'ye găhh

Ne gai'wa nehíak shăh;

Neh, kuh, neh ho'o weh, tăh'ăh

De'wăn dọh' ta'yos dáăh.

Benjamin Beddome. 
Pleading for mercy.

Da gi'dă nih, neh Swèn ni'yuh, We suȟ neh' sa da'ni dăos'hăh̆;

Ga wěn ni yuh' he'ni we suh'

Iis sa da'ni dăoss'yo wa năh.

2 Sah doht' neh de go'wă nyah'goh,

A gwv's' da gyah'do aih'jih', kuh, He'ni wáet gă̌h na gěn'yah są̆; A gwves da gyah'da goe'wah jih. 3 Tăh̆ ăh' deh̆ ga'dă no'wěnt hah, Do găs's a gi'wa neh'a goh;

Neh niih' a gi'wa neh̆'aks hăh Dyut gont neh o'ặn dọ' ga yăhh. 4 Wi yu' so nih' na gěn'yah sah, Iis neh Swěn ni'yuh he'go heh; Dak ni'goo nih neh tgai'wa yeī̌; Ih, dih, dă go'

Isaac Watts.

:PR

L. M.

Stay, thou insulted Spirit, stay.

O iis, Sat waih'chi yu, są̌ h'het, Sga gont' da gyohs' kni go áh gèh; Neb huh', naeh, sgoh'ni goănt'hwat hah, Oh ga'd $\mathrm{a} n$ do'nyaht' ne' swă nạh.

2 Să noh' wak ah' ă sga'do nyaht, Neň huh', naeh, niī o gă 'is doh A gi'wa neh'a go'on dyiěs; A ga'dye sah'dōh neh sknóoh g'wah. 
3 Theh్'wa dye'săh ae'sa ga'yăh A'sa díuh daat kni go ôăh gěh; A ğěn'nyah sah a'snăh na'wănt', Neh, kuh, o'sa ga'dat he wat.

4 Theh'o gwe'nyooh a'seh he'aak, “Do găs dăs khe'i wah'să gwưs: A skhe'yoh het ne găăo'yah gěh, Neh huh', naeh, goi'wa neh'a goh."

5 Do găs' ga we'e yoh'ji wăh No'gweh, gai'wa neȟ'ak shă go: Tis, sho, Seh has'des yo wa năh, Seh has'des hăăt, nă che' yoh het.

Charles Wesley.

\section{B:3}

\section{7.}

There are angels hovering round.

O năh' ho non'dặn da dyiěhs Na di o yah'ge o noh.

$2 \mathrm{O}$ năh' hăs ha di'wă a

He'o weh tho noh'dă dyo.

3 Don da'o noh dă ${ }^{\prime}$ dyoon dyiěh

Neh ho di'wa'neh̄ a goh;

4 Neh kuh neh Cha gáo he dvs

Ga g'we goh sha go'gwat hah;

5 Neh he ni yo'gweh da ge Do găss' jon dat'he wat hah;

6 Kuh ne Cha ga'olhe dvs

Do găs ho wai wa núvoh gowah. 
Seeking Christ.

Jat gat'huh he'ni sho'gwa wih,

Neh no něn'nyah si yu

Sha dih'ni goo 'ănt, sho, na'eh,

Neh no năhh' găóg'yah gěh.

2 Neh de'ă nan dah'no'oh gwah;

Neh neh Cha gao'he dv's

Neh ă sha'go nóoh gwak dyut gont'

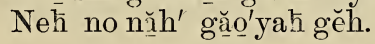

3 Iis, neh Sa yah'da ne'ă gwat Neh neh găa o yah gěh

Neh huh' niis sna geh'o weh, neh,

Kuh, neh so g'weh'dah̆ shoh.

4 Da neh huh', dih, a'se gon dak Neh niih a gat'wais'hăh:

Neh na yuh'ni go'i yu'ak $\mathrm{Ha}^{\prime} \mathrm{yu} \mathrm{i}^{\prime}$ wa da dyiĕh.

5 Neh huh' hă swa'ga dă'no dahk Neh ta'ga dó'is hăăh̆

Neh neh dă goăh'săá o'nyo ok

Neh neh ta'wa dohk'dăh. 


\section{THE CHRISTIAN.}

JUSTIFICATION AND ADOPTION.

\section{:}

\section{The joys of conversion.}

Do găas' ho'ni dắoh

Je sus ho want'hon deh;

Găógah ge'g'wa wa'o ne noon'dyiěh:

Daa'yont yuo wih̆' no'gweh,

He ni oh'ni goi'yuh

Neh wa'eh jago'dat he'wah doh.

2 He'ni wak'ni goi ${ }^{\prime} y u$

A ge'gă ne Je sus

Naat g'wăhh'sah nă wa'gyah da'ge hah:

Shah gi'wa g'wěn ni'yus,

Oh ni go'i yus deeh

O gyah'do wă̆nt a gěn'nyah saȟ'gěh.

3 Neh huh'. nakk ni'goă wăh,

Găo'yah gèh he gin̄'dyoh;

Wáe yăn deeh, kuh, ne Cha gaọ'he dus

Na dío yah'g'ge'o noh,

Neh det ha'sih da'geoh

Des ho wă̆h's šo nyoh', ho'da ni dăoh.

4. Neh he'ni wă'nĭs hes,

Je sus ho n’ón gwah'shăh

Dyut gont' neh a ga'dă no'dah goh.

Theh c'gwe nyo, ğăs, waī̄',

Ga g'we'goh a'ye gă

He'ni o da'ni dăos'yo wa năh! 
5 A gi'wa ne'ă gwưs,

He ni ak'no'oh gwak

Neh chi wa'gi wa'neh a'goon dyiěhs,

We suh' o gi'dăs thet,

A gyah'dah doh'o noh

Neh huh', gwa', naeh, ha gi'e yah seeh.

6 Oh g'eh'shă nih, a yăăh,

A gi'wa neh'ak shăh

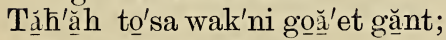

To'sa gi'wa neћ̆ aak;

To'sa wa'gyo ya'găak

Tăh'ạhh tọ'sa wak'ni go'da guh.

\section{Charles Wesley.}

\section{6}

\section{M.}

Peace and hope of the righteous.

Swěn ni'yùh', neh no'ni dạă oh',

Des ho wan diì'wah̆'să g'wah'seeh;

Oh dwa dă noo 'ya nit, gih shăh';

Sgă nōh' naeh, thiă nă noh'do nyoh.

2 Sgă noh, ne' wă nĭs'hă dee nyoh, Wa díăt hă haa di'yał daa deh;

Kuh no nàh oh wa'dă a nùs

Ooh'dă oh' sho, heh, wa díăt hah.

3 Os noo weh' ho di'yah doo wăhs', Neh, sgă nō h's', o noh'do nyoh'g'weeh; Ho nont'hwais'hăh shoh, dyót gont', naeh De yùs dăat heh', de yơ dạa ăh'gont.

4 Dyot gont sho, neh huh', ȟ́a di gat, Găo ${ }^{\prime} y a \hbar$ gěh' dyo non da dee nyoh'; Dyc̀t gont' deo non dặă nont neȟ huh', Heh, chih dyoh, hoo sa ìnih dyoo dațk. 
JUSTIFICATION AND ADOPTION.

5 Deh thah' g'wǐs dăh̆' ho nă'noh dô He ni yò dye ăh' yoăn'jah̆ gěh; Sgă noh̆', shọ, thiă nă nís'hi yaȟs, Thi a a 'noh son di yahss', gwah huh.

6 Neh' dih, he'noh ó g'găn dạă Aas'gwă dạăhh, aa ga noh'no wănt

No gwěn'nyah sah, ne Sat hwais hăh; Neh', sho, ha jo g'wa yoht' chih dyoh.

Isaac Watts.

\section{:8y}

\section{$6,7$.}

O brother, aren't you glad!

O de dyá dă no deeh',

O năh jih sah ni gói yuh,

O năh' jih sá duh sho

Neh deo nō dah'so dai goh,

Chorus-Neh Mo ses shá ne ga ek, Nok sah̆' dah shoh ho díăh doh, Neh Mo ses shá ne ga ek De yúh hne gúlh dah hoh.

20 de dyá dă no dee ${ }^{\prime}$ O năh', jih sa det gă deh,

O năh' sah nya găh oh Heo weh', ni sno sg'wa yă dvk.

Chorus.

3 O de dyá dă no deeh'

O năh' ja wă ${ }^{\prime}$ det gă deh',

O năh ${ }^{\prime}$ sah̆ seh' nya găat, Héo weh naeh sai wah'doh oh.

Chorus. 
CONSECRATION.

L. M.

What shall I render to my God?

O Swěn'ni yuh, Tis skno'oh g'wah,

Neh naeh, do găas o gi'dăs thet;

Tga gont' hă gyoȟs' heh sa noh'sòt,

Dặ găhh'săo'nyo ok̄ sah'să nâh.

2 Neh he'ni wa'gi wih'sah oh, Neh no năh' dwa gyo ${ }^{\prime} y a$ găs.s'oh, O năh' tga gont' ă gi'wa yéís He'a di gat sa haȟ'shăh shoh.

3 O năă'gwat he ni soh'hoh dặ;

We suh' she n'́oh gwah so gweh'dah;

Ji soh'he do'noh na gat'waih;

Dyut gont' ă goi'wa yă 'ni ak.

4 O năh oh ga'da dat gah', sho;

O ni yoh' neh kni gọ ăh gěh,

Ga do'gă hă wa ge noon dyiěh;

Ha'skhaah he yu'a do găh'doh.

5 Nǐk'huh ni ga'noh sòt, o năh'

Oh gi'wa gon duk he'goh heh

He'syah da deh; oh gi'wis aah

Dyut gont ăk ha'wak ne swă nạh.

6 Iis, dih, sgwa di'wat hon'da di,

Iis, neh Je sus ho'hah'shăh shōh,

Neh niis tga gont swai'wa gwaih'sih,

Dyă gwah' gih shăh' $\underline{a ̆}^{\prime} e^{\prime}$ yat gah. 
The counsel of His grace.

A gěn'noh dō ho heȟ'o weh

Neh Sha goh'he doh, daa'geh nyaah;

Sho doh'he doh na wée yo'noh;

Dya'wăhh oh', kuh, She'go wa'năh.

2 Sho heh' năs'ha'go yah'da guh;

Cha'go wĕn ni'yū̌ nceh tgăs shoh̆; Sho heh' năs'ha'go dăăh no'gweh,

Năs'hak'wa nont neh gut'waih.

3 Sho heȟ' năs'ha'gyah da'ge hah,

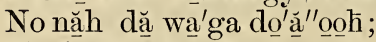

Sho heh' dăs hak'ní gonon'do nyooh

Neh nă wa ${ }^{\prime}$ ga dăh'swa et găăht.

4 Sho heh' năs hak'ni goă'na wănt

Nos'deh na gah'dyoh gwa'nīs thaћ;

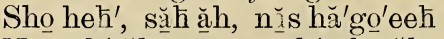

No gah'săh ne ge goh'sah gěh.

5 Sho heh năs'ho do gă $\mathrm{s}^{\prime}$ do ok

Na gén'yah sah̀ de yu'dya th koh;

Sho he⿳亠丷厂 năs hak nóoh g'waak' dyut gont',

He nă swa'gă nĭs'hă geh'het.

6 Sho heh' ne dăs̆s'he'no o nyooh,

Ăs'he ya'd na na $\underline{a}^{\prime} y \underline{a}$ ni'aak;

Sho heh ñ s hak'nok dv's'yo nyặh

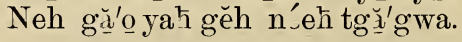

7 Ga g*we'goh, sŭh ăh, ne

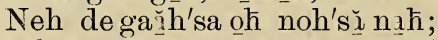

Náo hwah na'goh'he dớon dyiěhs,

Dya'wăh̆ ôh wạ nìs'hă de'nyôh. 
Mourning departed joys.

Oh'ni gó'i yus deeh' no năh'

Nih̆ shah̆ gắni nan dok

Neh de ja goi'wah să'gwat hah

Na'at g'wăhh'sah ne Christ.

2 Ao'wăh̆oh na gěn'yah sah్h'gěh No'sa ga'no a'eh

He'ni wa'et găh na gat waih',

Neh kuh, no'so go'het.

3 Se deh'ji ah, kuh noh găăs'ah,

Neh ga dă'no dah'gwah

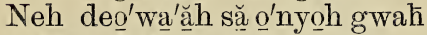

Na yah̄'da gwěn ni'yuh.

4 Neh, găa, ne'ya dă na'yă nih'

Neh Cha gáo he dus;

Neh ak ni gó'i yus dah'goh

He'ni oh'să noh'dă.

John Newton.

41

C. M.

For the return of the Spirit.

Gih saas ne da'yuh hat'he ak

Ho'a deh, neh neh huh'

He'yo aah̆'di oh, he'shăh dyoh

Neh Cha gáa he dus.

$2 \mathrm{He}^{\prime}$ ni wak'ni go'i yu'năh

Chi wa'e sha'e găh̆

Na yah'da gwěn ni'yuh! O'năh

$\mathrm{Ne}^{\prime}$ wah a gah'do niih! 
3 Don da'sah get, Sat wais'hi yu, Sa johs' na geèn'yah sảh;

Don da'sah get, o năh' săăh'ăh, Swa ga'dat he'wah doh.

4. Neh si'gwa he ni wak njóh goh Nìk'huh yo ăn'jah gěh, Is sa go 'yah̆da gwwěn ni'yus; Is, dih, a'sgye nă wăhs'.

william Cowper.

\section{Love to the Saviour.}

\section{7.}

Sat'hon dek', a gat wais'hăh, Hot haah' ne Cha gao'he dvs;

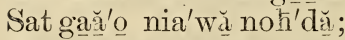

Yoeh'syoh g'wa nih nae'snúoh gwaak.

2 A g'wus' tho da'ni dăăs'soh', Iis! tya wă năăh'syoh, ha'doh "Gwe! Sa i'wa neh'a gooh',

O săn'dăs theht sknóoh g'wah', găah?

3 "Chi sa'yah dah'doh oon'dy̌̌̆hs Ih', waeh, sgọ yah'du'vs hă nyó ; Neh no năh' chis snos'gwa yăh Gơ dăăs's'oh, sgos'hăon da'gwăhh'.

4 "Chah'de sa dah'son dai goh

Iih de go'hat heh'da nii,

Gwe! sa i'wa neh'a gooh',

O sùn dăs'theȟt, sknoôh g'wah', găh ?"

William Cowper. 
Together let us sweetly live.

Do găa skă noh̄' hă dwa'na gek

Neh huh' wah̆o'gwe noon'dyiěh Ke'nyăn;

Do găă', gwah huh', hă ji'dwă eeh,

Neh huh' wah̄o'gwe noọn'dyiěh Ke'nyăn.

Chorus-O Ke'nyăn, neh Ke'nyăn,

Neh huh' waho'gwe noon'dyiěh Ke'nyăn,

O Ke'nyăn, nę̆ huh' dyo gwa'noh sòt

Neh huh' waho'g'we nọn'dyı̌ĕh Ke'nyăn.

2 Hä no'gweh di yu ho nuan'deh,

Neh huh' wah̄o'gwe noon'dy̌̌ě̆ Ke'nyăn;

Ja go'da'dwĕn ni yuh g'ua deh,

Neh huh' wah̆o'gwe noon'dyiěh Ke'nyăn.

Chorus-O Ke'nyăn, neh Ke'nyăn,

Neh huh' waho'gwe noon'dyiě̌ Ke'nyăn;

O Ke'nyăn, neh huh' dyo gwa'noh sơt'

Neh̆ huh' ${ }^{\prime}$ wah̆o'gwe noon'dyiěh̆ Ke'nyăn.

\section{ACंTIVITY.}

44

L. M.

Not ashamed of Jesus.

O gwe nyoo gọhh', a ga dešh'seek

Heh syah'daa deh, Iis' ne Je sus;

Tăh'ăh; t⿳⺈口̆ ăh' deh o'gwe nyooh',

Neh huh' noólwak ni gooh'dà a ak.

2 Iis, să ăh

Na gat'hwa is hăh; aa'gă noh̆,

Gih shăh', deh sa'yah da geh hăh,

A'yò dă.s'theh oh, naeh, ne neh. 


\section{ACTIVITY.}

3 Deh oi'wa yăs doh na'ga deăh,

Iis ne găh sa dyă nohk'dả oh He'yoăn ja deh, kuh, he goh heh; Iis, kuh, des găah 'nya dôh dyòt gont.

4 Deh oi wah'g'ěh na ga deăh seek; Tăh ăh, waeh, Niis; deh sa deăhoh, Oi wa neă'gwat ni ya'wăh oh, Sgie'yah seeh heh, de ga'yah sont. Joseph Grigg, alt. by B. Francis.

TRIAL, SUFFERING AND SUBMISSION.

45

How firm a foundation, ye saints of the Lord.

O ni yoh' ga găh'săh'goh, swai'wi yus'doh, Ga yan'dah goh neh swai'wa gwěn ni yuh'thah, Neh̆huh' gai waah wat'yuo wih na wắnah gěh A nạh ot' ho'sa ăh, naeh he'ni yăah se'?

2 'Să noh' ă sah'dyohk, Ih, săhhăhh, ăh neh'sek,

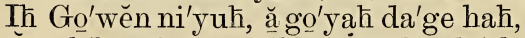
Á goh̄'has des'syo nih, dă goo das dó ok, Dă gớyah dah'gwah dah'go ok ges oh'dah.

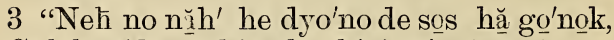
Găh han'de nyoh' neh oh'ni góă et gănt Tăh ăh de wăn doh' tae'sa yah'do weȟ'săh,

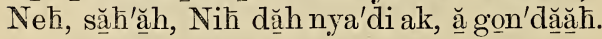

4 “'No năh' o jǐs'da go hă yut'hai'no ok, A ga'da ni dăos'hăh ă sa'yah'da guȟ; Neh noh doh'gwah tăh' Neh'sho ni wi, ă wà'des găh'să go o'eh."' 
TRIAL, SUFFERING AND SUBMISSION.

40

H. M.

The cross accepted.

Je sus, onăh' dă gěk

Neh neh de ga'yah sont;

Dyut gont' ă.k ha'wih sek,

Ä gónon dăă'dyiěh sek;

Ne⿳亠二, kuh, he'ni yoo' gyah dăăhs'gwah

O năhh' ga g'we'goh ă gat'gat gah'.

2 A gwa'nạh oh'dặh ăh

Ak'ni goì'yus dah'gwah

A gi'wa nósh goh, găs,

Nik'huh yo ăn'jah̆ gěh,

O năh, naeh, ă gi'wa gon dvk'

Oi'wa gwe'goh he'syah da deh.

3 A weh'oọn dyı̌ěh', shọ, naeh,

Á wa'gyah do'wăh sek

W̄a dăh'swa et'găh shăh,

A $w a^{\prime}$ gi dăs thełt't, kuh;

A gat'ga no ni, săh̆hăh, naeh;

Dwak nok'da yặh ne găăo'yah gěh.

4 Ā yơ ges swạăh' nóg'geh

A yok'noh gon dăh $\bar{h}^{\prime}$, kuh;

Á yơ'gat gaȟ', gīh shăh',

Tis ă sai'wah'gă ak;

Ak ni'goi yu', shọ, dyăa'gwah niis

Sah ni'goi yuh he'gyah da deh.

Henry F. Lyte. 
TRIAL, SUFFERING AND SUBMISSION.

48

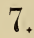

Jesus, Lover of my soul.

Je sus, snóoh gwah̆' na gat'waih,

Is ge'gwa hăs sga'deh gwat

No nŭh' ă dyo'no doon dyiěh,

Kuh neh ă dyu'doh dáon dyiěh;

Is, gwah huh, ă sgrah̄'dah set,

Neh ă yo'det gih'do ok;

A sgưs'de is n $\mathrm{n}$ go'hek,

Ăch hye'nạah na gat'wais hăh.

2 No'yah jih' deh dyo'nak dòt, Is' ge'gwa' sho, gyah da ahs', A'gă ak deh sgat'gah wăăh,

Dak ni góă ni yăăt'haak';

Iis, săhíăh, sgye'na wăh'seh,

Neh sho Nis geh has'deh goh;

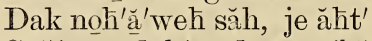

Snă'goos dah̆' o dăo nos'hăh.

Charles Wesley.

48

C. M.

When I can read my title clear.

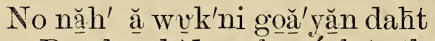

Dwak nok'da yăh néeh tgăh,

Å sga'gah chih kni goă 'et găȟs

Nǐk'huh yo ăn'jah gĕh.

2 Ă wv $\underline{u}^{\prime} g e \hbar$ shă'nyv's sek', gih shăh',

Na gi'wa neh̄'ak shăh,

Hă sga'deh gwat tha yah'da deh'

Neh Cha ga'o he divs. 
TRIAL, SUFFERING AND SUBMISSION.

3 A A wv'gyah do'wăh sek', gih shăh', Nă wakk'ni goă ạ $^{\prime}$ t gănt,

Neh huh' hă sgăh h'ni gọăg'gah'dăh

Neh gă

4 Neh săh'⿳ăăh, naeh, neh huh' tga yăh Noh ni go'i yus deeh,

Nih ă wvk'ni goi'yus dah'gook Hă yu'i wa da dyı̌ěh.

Isaac Watts.

49

Gently, Lord, O gently lead us.

$$
8,7 .
$$

Syăn ni yu, Syah'da gwěn ni yuh,

Neh niis ăs gwa'nă chi neh,

Ne yo ăn'jah gěh a gyoh'heh;

Da gyoh' sa da'ni d̆os hăh.

Iis a'sgwa ye na wăh'se aak,

He'ni sa da'ni dă oh.

2 O g'wa'yah do wăhs wa et'găh, Ti ga'wèn ni yuh' dwethah̆, Je sus tăhặh ta'sho g'waat gah;

Neh gwa', sho, nă sho'gvoh het.

Neh dyui'wah he sa'da no'thes

Ne Cha gáo he'da neeȟ.

3 Ah'soh sah̄'ni goă et găănt hah̆' Haȟ'de yooh ne yoăn'jă̄ gěh;

Ha yăn'neh goh hă ji'sa ah, Het'hăh dyoh Na'wěn ni yuh; Neh dyui'wah hesa'da no thes

Ne Cha gáo he'da neeh. 


\section{PRAYER AND PRAISE.}

5

I need Thee every hour.

$6,4,7$.

Dyut gont' iih aeh' neh sek, Neh Swěn ni'yuh;

Deh so gáh naeh̆ neh huh'

No yo gi dă.

Chorus. - De yo dọa juoh naeh neh sek,

Dyut'gont hés sgais da es;

O da'ga dăhh' swǐ yus'd

O năh, oh gèoh.

2 Dyut gont' iih aeh' neh sek, De dyá di ak

Tăh', thá gi wa neh ak

Neh ăh neh'sek.

Chorus.

3 Dyut gont' iih aeh' neh sek,

No năh'sgă noh

Ga jih' da gye nó wohs

Sạ nohtă gie.

Chorus.

4 Dyut gont' iih aeh' neh sek, Da gi' o nyăh,

Neh na' gi wa ye is

Sai' wiȟ sah'hoh.

Chorus.

5 Sa yah'da do'găh dih

Iis aeh' neh sek

Iis ă sa wă gă ok

Nih he'go heh.

Chorus. 
PRAYER AND PRAISE.

51

S. M.

The tender mercy of the Lord.

Neh des'she dwa no'nyo

Neh Sho gwa'wěn ni'yuh,

Neh săh̆ăh no da'ni dăos hăh

Neh tăh'ăh deȟ'o dohk.

2 Neh des'she dwa no'nyo

Neh Cho g'wěn'noh do nih,

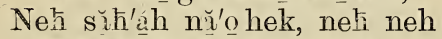

Tăh'ăh tha'wa dohk'dăh.

3 Neh des'she dwa no'nyo

Neh na o'hw it ge'ah

Neh no i'wa ne'á g'wah'dohs

He'ni o ye'ă noh.

4 Neh des'she dwa nónyo

Neh neh sho gwvs'hăăh'oh,

Neh nih neh wah̆o gwăn dăs'theht;

Neh chog gwa'yah da g'wăhh.

5 Neh des'she dwa no'nyo

Na yah'da g'wěn ni'yuћ,

Neh no da'ni dăos'yu wa'năh

Tăh'ăh tha'wa dohk'dăh.

Isaac Watts.

$5 \%$

$8,7,4$.

Seeking Christ.

Syah da'gwěn ni'yuh da g'wa yoȟs;

Tis' da g’wa'dăh swi'yus dăh.

Nă găa âk deh sa'ga yăh̆'ooh,

Ga g’we'goh ă gái wah'doh;

Da gwos'de is,

So hoh'ge ah sa g'we'nyooh. 
2 A'gă ạk deh sa'dō nooh' sho He'ni ya'g'wa yah'da deh;

Neȟ nă găą de⿳亠 sgowa'dyah dă niih Á ya'gowăeh, ă gai'wah doh;

Da gwus'de is, So hon̄'geah ăh'se g've'nih.

3 Neh g’wa', do găs a'ya'gwa nóok Nă ya g'wa'dă na yậáá,

Dyòt gont kuh da gowáăh săo nyo ok He ni yo ${ }^{\prime} g$ wă nis'hă gee;

Da gwv's'de is,

So hoh'ge ah sa g'we nyo.

4 Da gyoh' ne Sah ni'goh das'hăh, Kuh neh sat wai'shi yòs hăh;

Neћ ă yo'gwvs hăăh gwa'nis taak

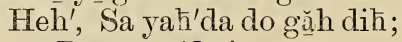

Da gwes'de is,

So hoh'ge ah sa g'we nyo.

58

$$
6,4,6 .
$$

Nearer, my God, to thee.

Dos găh' nih ah' dvk'his

Neh Swěn ni yuh!

Os deh̆' săh̆ăh ni ah

Niih sho dáak dat

Tga gont ș̄hăah sho', neh

A wa ga dă no dak

Dos găh nih ah dvk his

O Swèn ni yuh! 
PRAYER AND PRAISE.

2 A gyah'dah doh'ooh dy̌̌ěs

Oh so dá go

Oh'so da go gyas hăh

Neh gah sg'wăh̆' geh

Tga gont' diȟ'sho ne⿳亠 huh,

Neh ă ga dúok niih ah

Dos gah nih ah dvk his

O Swěn ni yuh!

3 Neh huh' o yă det het

N'et,găh ha geh,

Ga g'wé goh iik̄, sga wis

Sa da ni dă $\underline{\prime} \underline{\underline{o}}$

Ha diol'yah ge o noh'

De o ga dọa jo nih

Neh, dos găh no geh sek,

O Swĕn ni yuh!

4 O năh dă yoh'hat heh, Héo weh hă'geh, Ă s'gya găt' gá no oh

Aoh e sut kuh,

A weh̆ ooh dyiěn' ga nooh'

O năh', deh'sga no oh,

O năh săh̆ăh' ă h'neh

O Swěn ni yuh !

5 O năh' dă gá deh gwat

Găo'yah gěh gwa,

A wvk ni go hăh kuh

Neh yoăhi'jah geh,

Dos găh' ha gwah o năh

Héo weh hăs gih dyo dak,

Dos găoh' ah soh duk his

O Swěn ni yuh! 
PRAYER AND PRAISE.

(1)

The Lord's prayer.

Gwah̆ nih, ğ̌o ya ğěh chih dyoh,

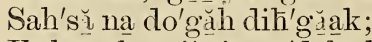

Kuh neh sai'wi yus'dō shăh

Ga yah̆'da g’wěn ni yuȟh heȟt.

2 He'ni di sah'ni góu ổ dă,

Naeh ni dya'wăs n'eh tğà'gwa,

Neñ huh', haeh̄'gwah, nậ'ya wăh

Nǐk'huh neh yo ăn'jah̆ gĕh.

3 Neh nă gă wă nı̌s'hă deh,

Da gwa'nont no gwa'ah gwah,

He'ni yó' swă nis'hăs hŏ̌

He'nia'g'wa ah̆'da na wăas.

4 Don'da g'wai'wah să g'wus', kuh,

No gowai'wa'neh ăk shăh shoh̆;

Dă jak hi'wah săgwus', kuh,

No khi'wa'neh a gih niih.

5 Să noh neh̆ huh' hă sg'wa'ah

Hă dyo'gwah ni'go da g'uh';

Don da'g'wa yah'da guȟ, gwa',

He'o weh wa et'găh gěh.

6 Neh ga yah'do weh'dus hăh, Neh, kuh, neh gah has'des hăh,

Neh, kuh, neh de gạăh'să oh

Is sa'wăh dyut gont. A men. 
PRAYER AND PRAISE.

55

C. M.

Thou dear Redeemer.

Iis neh snooh'gwah, Che yoh he dvs

Knoehs' nă wạ'ga to dek'

Sah să noh ak ni goi yưs tah,

Neh si'gwa o gah'oh.

20 dyut gont' a gat ho dek, niis Sa dani dăos'hăh othaa',

Tis syah da deh ă woga doh hook, Gih saas nă wạ go het.

3 Je sus dyut gont ă ga'do ok Neh'kuh yoă jah geh,

Âga dă no dăh̆h', kuh, o năh' naeh

Ga gwe goh ă wos ah.

4 No năh hăgs'gyoh, joh jih gạ dih, Ga gwe gôh nak nos deh Ás ga dă no duk', nak nos deh Je sus, sha gyah da g'wăhh.

John Cennick.

56

C. M.

Prayer is the breath of God in man.

Ăo dă nọ'yăcos Na wěn ni'yuh, No'gweh, neh, dăthah'get,

Ga nóọ gwah săh ă o yă dot, Âs gah̆doh go dăh' kuh.

2 Ă a oh' kuh deh júe nos deh, Nah ni goăh geh g'wah'huh. Dặs huh'ni gúo dặh na dạa nōhs, Hot chăh'doh ăo dois'hăă. 


\section{PRAYER AND PRAISE.}

3 No năh̆ Na wěn ni'yuh ăa ok, Elotho deh chi'wăh sah', Áa no et' nă ya'gùs sho dyăh, Âú wi yos yos da hah.

4 Neh ș̆h' heh go da ni dăs doh, As sha go ye na wăhs

Sạh' kuh, neh goi wa neh a goh,

Neh hawe yah'dah goh.

Benjamin Beddome.

ร.

C. M.

Prayer moves Omnipotence.

Neh nagah'shoh deh oh'dał was,

N'năh', oh dwa dah'so diik,

Neh kuh naódah dah déa swe goh;

IFo nẹh oh so da goo.

2 Neh na'năsah deh wa'chă tah,

O gweh ă wa chan naeh

Ho nooh gwah ș̆h thă tha gă ee

Yoă jah geh kaah gạ e yos.

3 Hot ga ă oh h'o weh hạss ha'dyăh .

Deoh gwah doh gạo ya deh,

Hoh to deh kuh no no dạ not

Ha n'ổ gwah shă neet găăăh.

4 Gah̆ has'des hah' ga yăh, og gweh'

Gowăh deh aoh no yah,

'Hya gạh tha nă sha deh, neh kuh

Neh ááok na ya go hek.

5 A dă nạ yă dvk săh heht găhh,

Je sus kuh huh sa noh,

Hah̆ dă ga'yăa nyoh', neh kuh ne

Ā ja go yah'da goh. 


\section{PRAYER AND PRAISE.}

\section{8}

I love to steal awhile away.

C. M.

Kno ehs' nă ga dă nos' gwat hak

Nă ga dă nó $\underline{y}^{\prime}$ ă nọh,

Á gat gas' kuh yaek ga g'we goh

Á găah ni gọă ni dăs.

2 Kno ehs' na'de gyah'dii hă geeh

Dă wa geh gah'săh dooh,

Háoh wọh geah sọo Na wén ni yuh

Áo tho deh'ji wă ok.

3 Kno ehs' neh no da ni dăos'hạh,

Á gă án $^{\prime}$ oh dónyoh gwak,

Ga gwe goh' na geh šh se'nehs,

Neh naeh ăa ${ }^{\prime} d y a \underline{a}$ no nih.

4 Kno ehs' hági wa gwă ni yòs toh,

We suh de yuh hat heh

A geh has deh sho nyah dah gwah

Heh nă yo nis het ageh sek.

5 Neh dih, no năh ă ket neh kuh

Neh ne teh'sa dye săh,

Tih sgăa noh jih šok nă chi neh

Ne deh odok a deh.

\section{9}

Mrs. Phœbe H. Brown.

Encouragements to pray.

7.

Agat'waih sa deh'shon nya'noo,

Jesus neh naeh yha nuoh gwah,

De ya'do ă jo nih iis,

Âh sah'doe syok tyă nos kuh.

2 Swěn ni'yuh o nạh oh gyoh', .

Ă ga dois'hăă da gye nạa,

Set gwăh sah swa gyah da gwăh

Tăhh ấh kuh tha wa gat gah. 


\section{PRAYER AND PRAISE.}

3 Heh, năayo'nĭs het ăh geh sek

Neh kuh sa nooh'gwah săh', neh

A woh'grah da do găas dook,

Hăk gwah is kuh he'yo dok.

4 Da go ${ }^{\prime}$ wothas heh nă gyeh

Ăk ni goăh'has dek gwah huh,

Ầ sa wă găok heh goh heh,

Âtis gieh ă so gweh dah'găolk.

John Newton.

(กำ

The pilyrims' song.

7.

Huk sah'dah shoh gowahh'go waa,

Dwat hai neh̀ dwa dạ no dăh,

Cha gao he dus de gaăh saoh

Noi oh dus hạh heh nio yeăhh.

2 Ha wă níyuh geh ho se dweh',

Hehs'shă nih dyoh eh tinok shoh

Tho dih ni goi yo neh huh,

Ji gwas' năhh ih hă dwa'găhh.

$3 \mathrm{O}$ ja doh ehs ho nyo ok

Christ deso gwa di wah nya nii;

Neh ne aeh'dyo hek i ga

D.̌ dwa'dăa no dạk gwah' huh.

4 Swat ges guh de yuh'hat eh;

Tga no da yăh öh swa găh

Deh o dok hă dwa no gek,

Āhshe dwa găhh neh huh ne Christ.

5 S

No nạh neh huh oh dyah'goh,

Je sus Christ, she dwanih kvh,

Ha dọ' huh swa dă dyo'dyiěh. 
PRAYER AND PRAISE.

(i) 1

$6,4,6$.

Jesus is mine.

Was ahs yoă jah ge chaa, Je sus tăh' naeh,

De wa dyahs no yah shoh,

Je sus tăh' naeh;

Oh so da go gih dyoh,

Deh aoh' a ga dois hă

Je sus haoh wah ge ah

Neh swa ga wăh.

2 Să noh ăsknii go da goh,

Je sus sho niih,

Dyut gont ă gih dyo dvk,

Je sus hạh dyoh̆

Gai wa dos naeh neh kuh,

Dah jiáh sho nă go hek,

Á gáat na gănn nyah sah,

Je sus sho niih.

3 Do sa gwa nọ'nyoh dih',

Je sus sho niih,

$\mathrm{Oh}^{\prime}$ so da go ił sweh̆s,

Je sus sho niih.

Ga gwe goh' na gat'waih

Oh wat'gat neh oh săt,

Je sus tuh̆ nih'goi yo

Neh swa ga wăh.'

40 năh' yoăh jah geh kaa,

Je sus săh hăh.

$\mathrm{Ha}^{\prime}$ ga wis deh o dok.

Je sus ho wăh. 
PRAYER AND PRAISE.

A ga wăh' hak'noōh gwah.

Neh kuh nă ga dois'hă,

Cha gao'he dus dyut gont,

Neh' swa ga wăh.

Mrs. Horatius Bonar.

(B)

$8,7$.

Come, thou Fount of every blessing.

Gajih' iis sadani dăa

Ses shọ nih na găn yah sah,

We suh' neh' sa da ni dăos'hăh

Na ga dă nu dvk nă iih,

Da gio nyăh nă Ho no dă not got gah deh.

Deo sa ă nyoh haeh gwah' nă iih, Sa nóō̄ gwah shă gọ ga dyă.

2 Nek'kuh, o năh', ă gat ges guh, Tis gwah huh' ăs' g'ye no wahs, Neh kuh' ni wi, sah ni goi yu, Sgăa noh̄' hăs gyah'teeh chih dyoh, Jesus ha gyah dih sas gwah, neh Chi wa gyah dah'doh o' dyehs, Neh no sa'gyah da guh' nuh'dyont, Wat gwăhh saih' neh na'go hek.

$3 \mathrm{O}$ niwe suh naeh a gat geot, Oh hăhh'sho nyoh oh dạ dyo, Neh dih', ne wi yo', neh, sa wặh'

A gas hăo'dăh na got'waih, Gyah dah dọs săh' geh gah ă yooh,

Akni go hăs syah da deh, Năăh' na got waih $\mathrm{O}$ sa je na, Ăh cheh săa no nih neh tgăh. 


\section{THE CHURCH.}

FELLOWSHIP AND UNITY.

\section{3}

\section{Love for Zion.}

S. M.

Je sus, Che yoh'he dv's,

Go, i'wa no'oh gwah.

Knúoh gwah no noh'sa do'găh dih,

Neh hiis sa dyăn'dah g'wah.

2 Dyus ah'doh khe núoh gwah Ne ga'wa ji'yă deh

O i'wăh gěh ho non'doh oh,

Iis she yah̆'da ni noh̆.

3 Tis săhh' set g'wăh'sa núoh .

Ji sa'no ăăh'dah goh

Nòt gih ne goi'wa neh ak shăh;

O năh' deh ja'o weh.

4 Dyă gwah' wăn doh', gih shăh', Khe yah'dah'ni go hăs,

Ga gwe'goh a'wak ni'goh hăh

Nak'ni goi'yus dah'goh.

5 Dyă gwah' wăn doh', gih shăh, Deh swa'ge ga yăs niih

Neh na'khe yah̆'da geh'hă ak

Deh gus'duk he'goh heh. 
FELLOWSHIP AND UNITY.

G4

C. M.

Christian band.

Găn dyò g'wah yăh o'ni dăh oh, ';

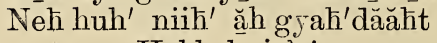

Hal le lu jah!

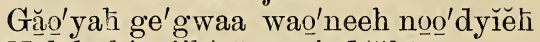

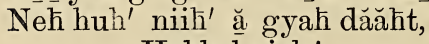

Hal le lu jah!

Hal le lu jah, Fal le lu jah,

Neh huh' niī̄a ă gyaȟ ${ }^{\prime}$ dăăht,

Hal le lu jah!

2 Hă nó goweh di yòhs gwah neh̆ huh,

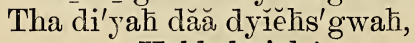

Hall le lu jah!

Ga grweh go' Je sus ho dyòh'gwah;

Neh huh' niih' ă gya dăăht,

Hal le lu jah!

Hal le lu jah, Hal le lu jah,

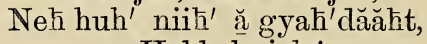

Hal le lu jah!

3 Da vid, gyeeh, ah yah'dăăhk, aeh gwah Neh huh' niih ă gyằ dăăht,

Hal le lu jalı!

Laz a rus, kuh, aeh gwaah yah' dăăhk;

Neh huh' niih' ă gyaȟ'dăăh̆t,

Hal le lu jah!

Hal le lu jah! Hal le lu jah !

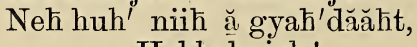

Hal le lu jah! 


\section{THE MINISTRY.}

65

C. M.

The Pastoral Office.

Cha gạo'he dus ho'haদ̌'shăh shoh

Ga g'we'goh ha di'yeh',

Ha di'ye nạah', hă nont'yuo wih

Neh o yah'gah hăos'hăăh.

2 Neh, dih, o năh', hah sa'ga ănt,

Na yah'da gwěn ni'yuh

Tga'ya gặht, ho di'yah do wăhht

Neh no non'di on dôh.

3 Neh nă găa nah̆ o'i oh'dăh

Tăhăh neh'huh deh neh

Ni yu'i wah̄aah hos déis doh

Ha gont'hă nih, i gă.

4 Neh gwa', naeh, hă on'di na het

Neh gă'o ya de'nyoh ${ }^{\prime}$

Ot wais'hăh'shoh, jo'yah da g*wăh

Neh gai'wah dưs'hă go.

5 Neh he'ni gai'oh dus'hă nóō,

Tăh ăh tah dáa di yeih,

He nio'dih ni'goh dv's'yuh dă,

Na dío yah̄'ge o noh̆.

6 Neh neh ot wais'hăh shoh dyut gont'

Hă yoh'he nyok n'́eh tgăhh,

Do gat na nis'he o'nah gèh

Hă yo'o ya'gă âk.

7 Je sus, he'ni sho gwa nóoh gwah,

Hot gah'wăh găo' yah gěh,

Neh no'sa yo $\underline{o}^{\prime}$ 'wa ya $\bar{h}^{\prime}$ da guh

Na nĭs'he o' nạ gĕh. 
(i):

L. M.

The Commission.

Swah'dăn dih, neh swai'o wa nănt

Na gi'wi yus'dôh shăh, ha wă

Na yah̆'da g’wén ni yuh, neh̆, kuh,

Yoăn'je g'we goh ha di'ye nạ.

2 Ă ${ }^{\prime}$ o hek' neh ă a $\underline{a}^{\prime} y a \hbar$ dáaht

Neh gwă năh; neh gwa', ga no'oh,

Heh nă ó ${ }^{\prime} y a h$ da wăh no no'gweh,

Neh tăhh ăh tai'wa gwěn ni'yus.

3 Neh Nih, o'yăn det' ă go'nih

Hah o i'o wa năh g'wai'ont;

Neh, kuh, o'yăan det' ă wan'dōh'

Gai'wa g'wěn ni yuh neh gwă nạh.

4 Neh ă wan dyeăh'dvk he'ni yo'

Ni wa'g'yăn na'wăh syōh, neh, kuh,

Ga g'we'goh o i'wa néă gwat

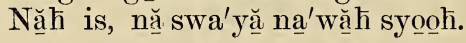

5 Swah'dăan dih, ă ye'chi jăht, kuh,

Neh go nok'da'no nih, neh, kuh,

Neh ă je'chi ges'gwa o nyooh

Neh o gweh, neh guh wăn'dah oh.

6 Swah'dăn dih, e chi'yah dit găh

Na di nis'he o năh, neh kuh,

Gwai'ont, să noh' ă swah'dyoh sek,

Dyă g'wah' ă ye chi găăăh'syo nih.

$7 \mathrm{Chi}^{\prime} \underline{0}$ nyăh na gi'wih sah'hoh;

Iih de dwa di' he'ni yoo weh'

Hă wan'do ăn'joh̄k dă

Naah'de ga gont' dạ gwăh'nya dook. 


\section{THE LOORD'S SUPPER. \\ COMMUNION.}

(8)

L. M.

Gratitude and Love.

O'gweh he ni ya'goh hoh'dă

Gạoh'es has, nă yon'da di dạăh

Neh'kuh, guh ni'go i yu, neh

Dyut gont' ă ya'go dặoh shăn dahk.

2 O we suh', gai'wa yăn'dah goh,

Nih, ă yo ${ }^{\prime}$ gwa dôh'es hă ${ }^{\prime}$ ak

Neh da'she dwa nóo nyo ok

Neh Je sus sho gwăa yah'seeh.

3 Neh dyu'i wah sho gwăe' $e^{\prime} y h^{\prime}$ seeh

Na'a nyuh no gwah'dyoh gwa nih;

Ă sho' gwah nya gănt' gă e'yat gěh,

Heh, de dyu dă nó ya nih'doh.

4 Neh nah'soh sho, hó'o ya găă

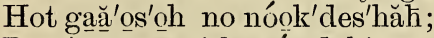

De o'gwe nyos'oh náa deh'gwus;

Neh nes'ga gont det hos'nyiěh oh,

5 Ha wăa', “dyut gont' a'swa yei'sek, Neh huh' nă gwa'yă noh'dạ a ak;

Iih' kuh dyut gont' ă sgowas hăăh'sek

He nạ ga'gas dặh yuăn'jă deh."

6 Neh nă gwas'hăăh'sek, săa 'e yoh,

O gwai'wa neh'ak shăh sa nyu',

Neh kuh, să a $^{\prime}$ ya găs'dah goh

Nih', he nạh'de gwa wă 'nyah khoh. 
68

C. M.

Surrendering to Christ in view of the Cross.

Oi'wa neă gwat he'nạ a $a^{\prime} w a ̆ h$

Je sus hot gwăh'saih'oh;

Neh, kuh, ho da'dat gah' 'wă nâh

Na'wěn ni'yuh, ă shăe $e^{\prime} y a h ~ d u k$

Năh iih o'sa gọ heek.

2 Neh, găh, na gi'wa neh'ak shăh Do găas' he'ni yu yéăh,

Hos syu'dyăs g'wah de ga'yah sont'

We suh, kuh, he nio'ya'găs doh,

Neh he'ni ak núoh g'wah?

$3 \mathrm{O}^{\prime}$ wa yăăs doh nạh ga yeeh Neh hoo weh ne găăh gwaah

Oh wa'dah sit noh son'da gọoh,

Oh gah'ho doọh o yăs'dos hăh,

Neh dah de ga ga'neeh;

4 No năh' hah has'dĭs yo wa năh

Ne Christ, Cha gao'he dus,

Neh huh' ha we'e yah'dah goh

No'g'weh go i'wa neh'ak shăh,

Héo weh de ga'yah sont.

5 Neh huh nă ya wăh, haeh'gwah, niih Å gat'goh sah sit, kuh,

Hah'de ge ga'neh ha yah thaah;

Oh tge'gah să'nĭs hăh'syoōh, kuh,

Niih he ni wa gyeă na

6 Deh o'gwe nyó, naeh, noh gah'săh

Neh a'wat ga nyahk'duk

Heh a gat'geot ha yah'da deh,

Neh̆ huh', sho', ni wa'ge gwe nyoo,

A ga'da daat gah'; sho. 


\title{
CHURCH WORK.
}

\author{
TEMPERANCE.
}

\section{(19)}

Tune; The drink that's in the drunkard's bowl, etc.

Ni yu'ne goh'dă no'ne gah

Nos'heh'de go i gaah

Ha'ne ga gah'wos thah'go waa

Tăh ăh .taa'kni ge'ăh:

Na'yah'dah, no'twaih kuk o nyus':

Ah hah'gih! gạa ăh, wa o'dặs thet

O dạăhht năa ${ }^{\prime}$ want gat huh !

$\mathrm{O}$ dă ăhat, năo ${ }^{\prime}$ want gat huh !

Tăh ăh taa'kne ge'ăh

Tăhh ăh taa'kne ge'ăh

2 Neh̄, gwa', Ne Găo yah gĕh Thăh'dyonh Sho gwa'ne gáa'non dăăh, Heh̆ tgăhh da oh's šh do on dyiěh;

Skăa noh no' sho gwa'non dạăh

o doh'hon doh no'ne ga no:

Aِ ga'gas dăh he dyoh he nyoh'

Neh', sho, ă dwa'ne g'éăh,-

Neh', shọ, ă dwa'ne g'căh:

Neh niih ă kne'ge haak,--

Neh niih ă kn ne'ge haak. 
90

C. M.

Tune; "Auld Lang Sine."

A gwáa wih a gyus'ho dah'gwah, Neh o yăn'deh dah'goh

A gwa'di yuh'dah gwah, neh, kuh,

Á yo'g'ga di'yu aak

O jis'dah nah o'ne góoh daah,

O gwa'deh sah'oh', kuh,

Á ja'g'wa da'dwěn ni yuh'het

Heo weh ya gwvs'hăon dvk.

2 Neh o gwa $\underline{a ̆}^{\prime} n a \underline{h}$ ă wan'do ok,

Neh niih o gwa'gah has

O'ne ga no o dos'ho doh,

Neh gwah' ti ga'na geh.

A gwa'ya soh, o'ne ga no

O gwus's'geh a ${ }^{\prime}$ geh dah;

Eh dah'gěh ă ya'gyo ni'aak

Neh ga ne'gah has deh.

3 Ha di'ne ge hah' no yăh'shoh

Neh brăn dy, beer, wine, kuh,

Hé ni yo weh' ' wáo di wă'naih:

Deh ja'goh ni gont', sho:

Neh niih ă ya'gwa yah̆'dăăhh'sek

Neh sho, o'ne ga no;

Neh, dyu'i wah a gwah'has deh

O gwa'doh ha eh' ${ }^{\prime}$, kuh.

4 Neh, dih, do'găs o weh o năh

Neh he" nah ó g' ga yeeh',

Neh wah'a gwa da'deh să năăh, To'sa ya gwa dyăăh'dvk 
No'gwe'nyo a'yo gwa'dya nok, $A^{\prime}$ yo gwăan'dăs thet', kuh, A'yo gwai'wah'doht he'dyo heh Hặ yu'i wa da dyiĕh.

81 L. M.

Jesus shall reign.

Ne sgáa hwah'soh neh he ni wehs $\mathrm{Ne}$ găăh'gwaaћ năg'dek haah, neh kuh Neh ha di'na ge nyoh' no gweh, Je sus ă sha'go wền ni'yuk.

2 Hao hwạh ho yah'do weh'dus hăh Ga gwe'goh neh dwănn noh'dos oh: Ga nyu'dai nyoh, yoăn'ja de nyoh. Nă no'gweh haa'di na ge nyoh.

3 Ă wan'dohk dăh ne găăh'gwě dyiěhs: Kuh haeh'gwah neh hes sgaa'hwah soh; Neh gwaah', nă yu'i wa da dy̌ěh Je sus dyòt gont' ăant hăhn'noh dóokk.

4 Dyòt gont' ho wann'dă na yă nih, Dyòt gont'ho wạn'dă no'da nih, Neh de o wạăh'să o'nyoh gwah Noh dye wạ noh'dăh shoh' no ${ }^{\prime}$ gweh.

5 Yek sah'shoh oh' ha di'găh jih, Ni oh'să no'neă gwat dă yek; No'núōh gwah'shăh ă yỉs'nyĩeh dvk: Dyòt gont hă'nan dọ es ho nyooh. 
92

Missionary hymn.

Neh huh' năn dyǐĕh'g'wa ho'weh,

Deh găăh'gwăăh' go ' gwa', kuh,

Ho ne'git găăh'syo nyon dyiěh,

Oh ne'gah dặn'dyo gwăh, We ăh'h' dyo ăn'ja de nyoh̆,

- Yo ăn'ja gwe'goh', kuh,

Dyo khi'nos na'yet hi'yoh

Neh gai'wi yus'dos hăh.

2 Héo weh' tha di'na ge nyoh

Neh dya wăn'det gă deh;

He ni wah dai'yah thah, kuh,

Neh dyuh'ni goil'yus deh;

Neћ̆ g'wa', na'di na ge'nyoh

Oh son'da go hă nehs;

O geweh'sho ho dis'yo ni

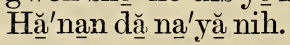

3 Neȟ'huh, dih' dwa'na geh

De yuh'hat hes'hă gọ,

Cha gaó he dv's sho g'wa'wii

Neeh tgăa ${ }^{\prime}$ gwa dyuh'dặn dyoo;

Á yet'hi vah'seh dăh', găhh, No ni'dăs theh'syo nyoh,

Na nĭs'he ó ${ }^{\prime}$ noh ge ${ }^{\prime}$ g'wa

Wa'o ne nó on dyiě ?

4 Hă yógwa aah' ne găă'hah, No doh'da on dyiěhs's , kuh,

Neh neh huh', dih, hae'dwa yoh'

No ni'e yónon dyǐĕh; 


\section{GHURCH WORK.}

Nehi' na'yet hi da gwaih'săh,

Neh Je sus ho gwe'nyo

No'sas ha gaog'he da'noh

Neh goi wa neh'a goh.

Reginald Heber.

78

L. M.

Hark, the song of jubilee.

Sa dáoh di yus, dyo'ă no gaah,

Ot gáeh nii, tga'wănno dahs', ăăh̆h

A yăăh o nyu'dăă gaah', neh huh'

Ga yăhnt hah he'yoăhn'da dyı̌ěh.

2 Des she dwáăh săoh Na wĕn ni yuh;

Gah has'des hăh neh huh' o dyóọh,

Neh ga gwe'goh ănt hă noh doook'

Neh' yoăn'jah gĕh, kuh' găao' yah gěh.

3 Swa dă ${ }^{\prime}$ nont hon daat:-a yăăăh', sho,

Ga gwe'goh' he yơ'non dạa a nòòt'

O dyeă'no nyoh heh' yoăn ja deh,

Neh kuh heh gă ó ya dee nyoh.

4 Shoh' să noo niih' noo su'dah gwah, Kuh nò̀h'sha ah;-neh no snyeh'oh, Oh wa'dah gwǐh! O năh' Je sus Neh sa'ăn ja gwěn ni yuh'heht.

5 Haah'de yo doăn johk'doh, Je sus

Oi'wa g'we goh' ănt hăánoh dook:

Neh' sho ăut hă 'noh dook', no năh' A want'gah chih găáo'ya de nyoh. 


\section{TIME AND ETERNITY. \\ WATCH NIGHT AND NEW YEAR.}

84

I would not live alway.

Sha yah'daat, neh no yah'do weh'dus hi yu, Neh huh' hoh noh'so niih neh gah sdạa ăh gě̀; 'Tăh', kuh, deh o'hnye năh'ooh hoh géo wa nănt Neh, săh'ăh, heh găh sdăăh's'gěh ga noh'so niih.

2 Sha yah'daat, neh ne de oh'ni gont no'gweh Gah neh'sah gě, sho, neh huh' hoh noh'son niih; Da ga'gon da dyeet, neh huh' ga ne'ga yănt. De yu'no ya nit neȟ'huh hoh gaȟ'hnye nănt.

3 Ga'ạa, noo, ni a'yah daah' a gat'waǐs'hăh, De ji'snya dye'ăh niis, nă gă dei'yvs heh ? O nắh', shọ, kuh, neћ̆ de jah̆'do weh'da noôh, Hah'soh deh sa'yah do wăhhs' ne ga no'ôh.

4 Neh waeh, ho nont'gah̆ deh ha dih'ni go ${ }^{\prime a ̆ h}$ Nă o dih'ni gó i yuh neh găọo'yah gěh; Neh, gwa', naeh, no năh' hă ${ }^{\prime a ̆ ~ n o n t ~ g a t ' ~ w a h ~ s o o h ~}$

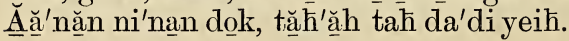

$5 \mathrm{Da}^{\prime}$ wă nok ${ }^{\prime}$, shọ, dyă g'wah ănt ga'gon da dyet Hă ga'ne ga yănt, ne' ho di'noh so niih Ă gah'hnye nănt: neh̆, kuh, de yu'no ya nit Ăo'di yah'da wăh'sek hă yui'wa da dyǐeh.

6 Neh ne'yu dohk ne g'wah ti'shă neh' no năh' Hă ga'eh neh hoh dwă'nĭs hă'gon da dyiěh. Iis, Je sus, da gye'na wahs, nek̆ tga gont' niih A'wa gat'hon dek, a'gyeis', kuh, neh' swă nạh, 
TIME AND ETERNITY--HEA VEN.

$857,6,7$.

The better portion.

Dis daht, O a gat'wais hăh,

Neh de sek ne wi yn,

Sat gah̆ neh yo ăn'jah gěh, Sat'gon dvk' găo ${ }^{\prime}$ yah gĕh;

Găăh gwaah̆, ga jih'soh dăo nyoh,

To hah', naeh, ă gai'wah'doh:

Do'sas daht', dih, a gat'waih,

Sat'gon dvk găo yah gĕh.

2 Găh han'de noh neh huh', naeh, Jo nont'gon dah'goăn dyiěh Tga nyu'de o wa năs' gwa:
O jĭs'dah heh tgă h', naeh,

Wat gon'dah gwah; neh huh', dih,

No twais'hăh, jo'na'gă doh

Ni yuh'dă, găo' yah gěh', naeh,
A jot'gon dah'goan dyiěh.

3 Swăa'ni hăăh, ne swa dăănahs',

Is, neh swai'wa yeis doh';

Neh săh ăh, ta'yo nis heh̄t',

Cha gago'he dv's dănt heh;

Ho dặh shă nyogo găo $\underline{o}^{\prime}$ yah gĕh;

Neh huh̄' tgăn dyuh'gwa yăh, naeh,

Deh'je găh no nook des'hăh:

Dyòt gont' sho go dă ${ }^{\prime} \underline{o h}$.

Robert Seagrave. 
BREVITY AND UNCERTAINTY OF LIFE.

96 $8,7$.

My days are gliding swiftly by.

Os'noo weh' o di'ăh doon dyiěh

Neh na găánĭs hă de nyoh̆;

Neh gwa' deh wiih' ă yuăh heht,

He ni yuh'dạa dyóon dyiěh.

CHORUS.

Neh săhh'ăh neh huh' dwah si'aah

Neh găhh'hakk ah' ne Jor dan;

Dea'di ya'yahis dwa dặ'nok shoh;

Dus gạooh eh thi'non dăă dyiěh.

2 Neh sặh' sho gwah ni gooh'dăa thah Neh neh Sho gwa'wěn ni'yuh, Tga gont' ă yuȟ̉'doh go'da dy̌ěh Ga gwe'goh dwa as'hă a a

CHORUS.

O năh' sặh'ăh he dwah'si aah Găh hạk'da dy̌ěh' ne Jor dan:

To hah' o g'we'nyoo ae'dwa găh Ke nyăn, neh sgặh'hạn di'gwa.

3 Swa di'gwa ho doh da on dyiěhss', $\mathrm{O}^{\prime}$ gwe nyoo' kuh ae'dwa gặh, Neh, he'o weh' hăgn dwăah'dyon dvk, Je sus ha yăn'neh gọ 'gwa.

CHORUS.

Neh săhh ăh neh huh' dwah si aah

Neh găhh'hakk ah' ne Jor dan; Dea'di ya yah̄s dwa dă'nok shoȟ: Dus gạooh' eh thi'non dăă dy̌ěh. 
DEATH AND RESURRECTION.

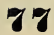

C. M.

We mourn not as those without hope.

A naeh' go waah' et hih ha dạăhh, Sa yok' hi yah'don diih ?

A gwv's' wah o gwa yah don daaht, Găe yaht' waa'chỉh gwaa thòh.

2 Neh waeh', sho, ne Je sus daas nyeht Neh don daa da'nii nook

Hah dặs'hă non'di aak' o năh,

$\mathrm{He}^{\prime}$ dyō̄i ni goi yòs dee⿳亠丷.

3 Tăh ăh, găh, nạăh̆h' iih heht gạă 'gwaa Theh dwah'ni gooăăh̆, hae dweeh ?

Să noh' dih, ă yo gwa noh dohk, Ji gwevs' sho hăn dwaa yoh.

4 O gwa yah'don dah'daa neh' gwess, Wah et hih sa'doo neh:

Jis sas'hăăht sho, neћ̆ huh' haeh'gwah, Hea'yas hăn duk Je sus.

Isaac Watts.

78

L. M.

Christ's presence makes dying easy.

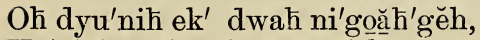
$\mathrm{He}^{\prime}$ ni dwas'ha nis ă dwă ${ }^{\prime} \mathrm{eh}$;

He nạho'gwăn dặs thet' ne dyo' g $^{\prime} w e h$, Dwa nưs'deh̆ o gwặo'ya gặs'hăhh.

2 Neh o nóok des'hăh, gus'syo dyăs', Go ó ya gă ne yai'ye yos, Neh̆, găs, o gwah'dyoh gwa'nĭs thah, Di dwa'dō nes ne gặe'yaht gěh. 
DEATH AND RESURRECTION.

3 Neh sah gwah', sho, dwa da'dă gwvos Nae'dwah ho dok', shọ, yoăn'jah gěh;

Neh, naeh, i dweeh' ne găa'e yaht Gah ho gaă n'dah goh tgạao'yah gěh.

4 Neh gwa' dyăa'gwah Cha gaó'he dus

Ầ 'ya kneh', a gat'wa is'hăh

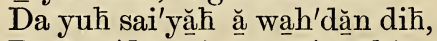

Dạ wạn'do gut ne găa'e yaht.

Isaac Watts.

7\$

Friend after friend departs.

$6,8,8$.

Ho di'ăh dọ'on dyiě

O gwa dăoh'shoh găáăh;

Deh gai'wa'gas deh he'dyo heh

Nik huh yo ăn'jah gěh;

Neh gai'o nih deh os'haeh'gweeh

Nǐk huh a a'yo gwa dois'hăn dahk.

2 Si gwa nặ wann'dih gěh

Dyon da'deh sa'doh gwah,

Neh ho'o weh dyo ăn'ja deh

Neh oh ni goi'yus deeh;

I dwe', neh huh' sha di'na geh

Yoh heh'o weh sho non'doh̆ oh.

3 Ta gai'wis heћt, o năh

Iih, kuh, ă dwat gat huh

Cha go'des ah'seeh ne Je sus

Hao hwah' ha o'gweh dah;

Iih, kuh, do găa ăn ji'dwa dōh

Neh̆ nìk nuh o khi'yah don dyooh.

James Montgomery. 
DEATH AND RESURRECTION.

81)

C. M.

$A$ voice from the tombs.

Swat'hon dek' dyiut haah' he'o weh

Dyon da'deh sa'doh gwah;

“Tis, neh jo' gweh, ta'yo nis'het

Nik'huh hăh swa'dyvs hăăh.

2 'Tis, kuh, neh swa yah'da núo nyoh,

Neh swah ni'go wa năs,

Nǐk'huh ă swa'yah daăh heh̄'syooh,

Shaȟ'dặs swa yah'da wăhh."

3 Sa yaȟ'da néă gwat, Swěn ni'yuh,

Neh̆ huh' ni ya'wăh seh?

Neh no gwa'dyah̆ dv's'hah oon dyı̌ĕh,

Deh o' g'wa deh'sah hoh ?

4 Da gyoh', neh, o gwat'waĭs'hăh'shoh

A'gah has'deh go'ok.

No năhh' ă yo gwăe e'yo on dy̌̌̌̌h'

Na ya gwah'săn'no nih.

Isaac Watts.

\section{JUDGMENT.}

81

C. P. M.

Supplication.

O Tis, ne Sai'wa ye'is doh,

Ne de sgyah'do wet hah, no nă h'

Dănt cheh', ă che'nok heh

So gweh'dah, she yoh'he da'noh;-

No năhh' heh tgăhh' ă she'don duk,

Neh, găh, nă gyah'dăă dyiěh ? 


\section{JUDGMENT.}

20 gwe'nyo neh huh' hah'daak daht' Iih, neh sgye'ăh no jih'no wăh, Iih, neh deh tah'gwis dăh, Tih. neh gěs ha'nis na'gi eeh, O gwe'nyo, găah, hă gih'dyon dahk Neh niis syă neh'go'gwa?

3 Gi wa'no ehs ne'wah, nǐk huh, Da'ya gwa'di aak so g'weh'dah, A'ya gwat gěn nis'aah, Do gă a $a^{\prime} y a$ gwa'dă na yăăh̆ Neh huh', naeh, niih o gi dăs theht A gi'wa neh'a goh.

$4 \mathrm{O}$ gwe'nyo, găah, na'gat gwe nih', He'ni găoo' ya'găs yo wa năhh A'găn noh'do nyoh'gwaak,

Neh' ${ }^{\prime}$ gwah, neh oh sga'dah sănt niih Năăăga'eeh nănt che'yah déo ek Ga gwe'goh so gweh'dah.

5 O a'găa ak niih deh'sga dyooh'!

$O$ iis, Swěn ni'yuh, a'sgi dạăh̆, No năh' hă wă 'nis hăeh, A'ga dah'set heh syah'da deh:

O a'gă a de deh sgat'gah wăăh ! A'sgyah dah' să no nih.

6 No năh' dă dwă $\underline{a}^{\prime} \underline{h}$ da'do akk, Dă dyont'găă neh guh wăn'dah oh, O niih a'sknok do thes Ha'gyah dă dyǐ̌̆ sek so g'weh'dah, Dyut gont ă yo gwa'dạ no dahk, Dă gwạăh'săa o'nyo ok. 


\section{HEAVEN.}

\section{M.}

The prospect joyous.

No năh' gyah dah' ă dyuh'syo nănt,

Ă ga'da gắ ${ }^{\prime} \mathrm{ehi}^{\prime}, \mathrm{kuh}$,

Hặk nok'do wặht ăa'ga doiss'hăăh,

Ne Je sus het'hăh dyōh.

Neh, naeh, skă nō̄' gén noh'do nyoh,

Ah'soh nik'huh i gehs,

$\mathrm{He}^{\prime}$ o weh oh'ni goă'et gạnt,

A gyo'ya găa'an dyiěhs.

2 De ga'yah sont a a kha'wih sek, He ni yúo weh' dănt heh'

Ne Je sus; ă sha'gyah da guh,

Hăs'ha khaah' het'hăh dyoh.

Gáă gwah' ni yu'i wa'neă gwat

Je sus hak ni'nă seeh;

Găh hann'de nyoh yoh heh'o weh

A ge'gă găăo'yah gěh.

3 Deh thah'gwis dăah, ha genn'noh doh, A gyóya găs'dah gôh,

Dyă gwah, gih shăh' hah'de gye'ih

'Neh niih neh huh' ha gyoȟ'.

$\mathrm{O}$ iis, seh has'des yo wa năh,

A'sknok do thus' niih ah

$\mathrm{Ha}^{\prime}$ gat gat huh' de gaăh'sa oh

$\mathrm{He}^{\prime} \mathrm{o}$ weh niis chih dyoh. 


\section{HEA VEN.}

8:3

\section{The home over there.}

O să noh'do nyo hăs no gek, Neh huh tgăhh ho deh de yo'hat eh Tho di wa yeis doh he sho di yo,

Ho noh sho ni kuh ga găa ăt.

||:Găao'yăh geh, gạăo'yăh geh,

O să noh'do nyo hăs no gek. :

2 She yă noh'do nyo she nok soh Ho diăh'doh o ă do jo dō ôh, Neh huh naeh kuh he sho no dă not

He thăh dyô̆ neh Ha wěn ni'yuh,

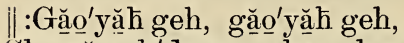

She yă noh'do nyo she nok soh. : :\|

3 Cha gao'he dv's neh huh hes'hehs, Khe nok shoh neh huh ho no'doìs hăh

O năh dih ă gat'gah neh oh săt

O năh neh huh ăhs gat'go dvk.

\|l:Găago yăh̆ geh, găăo' yăh geh,

Cha găón he dus neh huh hes hehs:\|

\section{0 năh tho hah nă îh hăs gyoh,}

O năh săh̆ăh neh huh he ge găah,

Got gah deh khe nok shoh' ne neh'huh,

Tha dih ni góăh neh hặs gyoh.

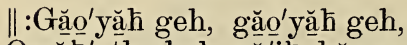

O năh' tho hah nă ${ }^{\prime}$ ih hăs gyoh. :\|

D. W. C. Huntington. 
We are on our journey home.

O năh' jo gwah'dă dyoon'dyiěh

$\mathrm{He}^{\prime}$ gwa hes' ha we noo

Neh Christ sho'gwa wěn ni yuh';

Neh huh' hă 'jo gwa ye ih'

Neh dwa seeh, neh dwa seeћ Je ru sa lem.

2 He'dwa găah dyo gwa'noh sòt,

Deh gai'wah hoh jih'geh;

Dwa găh' ga noh'sa gă $\underline{a}^{\prime}$ yaat

De dwa dăa ăh gon do nyoh

Neh dwa seeh, neh dwa seeh Je ru sa lem.

3 De yuh'hat hes'yo wa năh,

Neh tăh'ăh deh găăh'gwăhs;

To hah' ăn dwa do is'hăăh,

Neh huh' hăa'jógwa ye ih,

Heh dwa seeh, heh dwa seeh Je ru sa lem.

\section{O! dyo nok'da do'găh dih}

Cho gwa'gă ya da ni

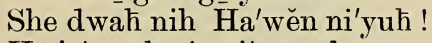

Hă jo'g'wah ni goi ${ }^{\prime} y u$ aak

Heh' dwa seeh, heh dwa seeh Je ru sa lem.

5 Iis neh Syah'da gwěn ni yuh,

Oh gwa'do e'syoh gwăh,

Don da'gwah ni'goăn de nih,

Neh huh' ho'sa ya gwa yoh'

Neh dwa seeh', neh dwa seeh' Je ru sa lem. 
The heavenly Canaun.

Si'gwa dyon da'deh sa doh gwah, Neh huh' dyoăn'ja deh̆;

Thă nih'dyoh no dí'wa véis doh, Heh dyuh'ni goi'yus deeh.

2 Deh aoh' a'ya go'yah do wăhht, Neh oh ni goă 'et gaăănt,

Ha go'yv's deis'doh ne Je sus;

Dyut gont' deo wặh'săao nyoh.

30 ! ă yo gwah'ni goi yu'aak Dyă gwah' iih ai'dwa găhh, A'găa ak deh o' gwah săh'seh No năh' dănt sgowă'nạk heh.

4 Da gwa'ye na wăhs, Swěn ni'yuh A $^{\prime}$ yo gwa'deh sah'ook' :

Neh niih' ' a'yo gyoh'es hah', kuh, Niis' heh cha dyăn'dah gwah. 


\section{DOXOLOGIES.}

\section{M.}

Blessedness of God's servants.

Ga gwe'goh neh dwa na'ge nyoh'

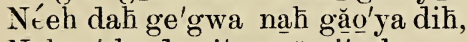
Neh a'she dwai'wa yă ni'aak Je sus, sho g'wa'yah dih'sah oh.

2 Ho da'ni dắos yo wa năh:

Nă yui'wa da dyiê̌h' go dặ'oh, Do găs's' no wại'wa gwèn ni'yus: Ye i'wă ạah', kuh, na'wă nạh.

\section{M.}

Pleading for mercy.

Wi yu' so nih' na gěn yah sah, Iis neh Swĕn ni'yuh he'go heћ; Dak ni'goo nih neh tgai'wa yeih: Ih, dih, dạ go ăăh să o nyooh.

:3

L. M.

Calling for mercy.

O Is, neh Syah'da gwěn ni'yuh, Neh neh gah has'deh neh snăs haћ, Os deh' he ga'eh gyah dah'gě̀; Is gạa âk shă guh, da'gi dặ.

20 Iis, neh Syah'da gwěn ni'yuh, Da'gye na wăhs ne waȟ t tặh ăh Deh a gyặh na' yo gye'na wăhs, Nǐk huh heh dah̆'gèh yuăn'jah̆ gěh'. 



\title{
$\mathrm{GA} \cdot \mathrm{YA} \cdot \mathrm{DO} \cdot \mathrm{SHA} \cdot \mathrm{GO}$
}

\author{
NEH
}

PSALMS

NE

\section{DA VII)}




\section{O I'WAH SKAِT. (FIRST PSALM.)}

f OD

f ha weh nooh dyiěh neh tăh 'ăh da wěn ni'yuh, kaah nă gă ok kuh neh huh dea'dăndyiěhs hea di got no di wa neh a goh', tăh'ăh kuh neh huh dea dyă'do nehs hă no dyă dahgwah na dih sga hah'thah.

2 Neh gwa huh ni goi'yus dah goh' no yanes ha go Na ya'da gwèn ni'yoh'; neh kuh noya nes hạ go ho de yặhs'dôh wạa nis'hă de nyôh wah sóda de nyoh kuh.

3 Neh naeh de sga dye ăh ha yah'da deh neh ga it ga yă'twah oh dos'găh he ni yoh' hne gah dă dyo no ne ga nus, neh wa da'yo nih ha ya'da dyiě̌ no năh' he sgaes; neh kuh no năhh'don ha yah' da deh tha ga da găe; neh kuh na di'gwa nah' ot năo'dyiěh ă wada wi yus.

4 Neh gwa neh hăh'ăh deh neh na wěn nǐ'yoh ka tăh'ăh naeh deh neh huh' deh oh dă : neh naeh shah'gat no gae'dah ạ tgaăh o yahgih naeh hă gaah.

5 Da neh dih' gạago'nih neh tă̆h'ăh da wěnni'yoh kaa deh aoh a ga dok' sho no năh' dạ swa'dyah do'wet, kuh no di wa neh'a goh ne neh huh' a di'yah daak heo'weh ă gă'dyugwa yă 'dok neh tho di wa yeìs'doh.

6 Neh săh'ặh Na ya da gwĕn ni'yoh ho nodôh ji'wăh he ya go tai'noo neh dyiěti wa yeis: neh gwa he yot hai'no neh tăh'ăh da wĕn ni'yoh ka neh naeh nă gai'wah dôh. 


\section{O I'WAH DEK NI'. (PSALM II.)}

A NA' EH GO WA neh deo noh dah so dai'gohoh, neh kuh nă nọ'gweh hă noht gaă ${ }^{\prime}$ oh neh deh wa des tăh ?

2 Neh ne go wăh go wa shoh ho no da dyah da gwĕn ni'yos doh nă gă heh yoă ja deh, kuh neh thă nă noh do ô ga do gă ha dia'shă donyoh, oh thă no do găant Na yah da gwĕn ni'yuh neh kuh ne Cha gaó'he dis's, hă nó'doh,

3 De dwa'yak gă ok neh do nă nih dyuhgwah has doh dă dwa kah so goh, ă yo gwa dih kuh, neh, da di'noh so dah'goh he ni dwa yah' da deh.

4 Neh ne thăh dyoh he găo'yah geh ăosgat gwăh: neh Na yah'da gwèn ni'yuh hă ${ }^{\prime}$ goh dyooh he a goh dyò dah gwah neh de ăno'tho das.

5 Da, o nặh a a as nyěet ha di yah'da deh

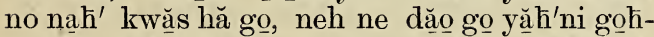
ga gat heh ni oh ni goắno os heh deht huh ni goi'yuh.

6 Neh huh g'wa'naeh nii heh dyoh ne gowăh go wa no nóda do gặh dih geh heh ó noda deh neh Zion na ga wăhh.

7 Tga gont' niih o yắdet ă go nih heh nuiwá ni yă toh: neh Na yah'da gwěn ni'yuh ha găa ${ }^{\prime} \underline{o}$ see, iis neh go o wak: nă ${ }^{\prime} g \underline{a}$ ă wăs'hădeh o gyă 'dot nă wa do nih heh syah'da deh.

8 Ăhs ga doe syoh gwăh, neh kuh ne tgagont' a a tgo yoh neh deo noh dah so dai goh 


\section{PSALM II. PSALM III.}

neh ăh syah da g'wěn ni'yuh'het neh kuh hehnoh de yo dọăjok da dyiĕh' neh iis ăh syah da g’wěn ni'yuh het.

9 Neh dă she yak dặh ăh syeăh dvk ne sa joh'dyah neh ga nyoh oh shăh : ăh she yă $t^{\prime}$ dạ gáit he ni ya wăs no gáhs dăh ga jặh.

10 Neh dih gai'o nih ja dạ ni go wa nạ do ok $O$ iis ne go wăh go wa shok: neh kuh ne swa de yăs, iis neh de swa yah do wet hah ne yoă jah geh.

11 He ji wa yă ni ak Na ya da gwwěn ni'yuh ă eh ses ha nih sek kuh, sa doh'ho ok neh kuh ne sa ya'do da tak.

12 De sa gwa yos no wa wạk, dyă gwah. săh'ăh ăo noh gwăh, neh hưh kuh ă gai wa do hă'chat hai neh, no năh' no năh'kwăs hăh osthóoh ă dwa chăo ne dăh. Ho ni dă neh huh heo di yă na di yah da as ha ya'da de.

\section{O I'WAH SÄH. (PSALM III.)}

¿HA DA GWĚN NI' YUIr, he ni gă h dyuh go wa nạh neh deoh ga.do ă o g'wah da'nih̆! got gah deh neh deo di dah oo dyiěh neh dă ya g'wa do gănnt.

2 Got gah deh neh hă năoh seh na gat waihăh, tăăh'ăh deh ga yăh (na oh. selah.) ye noh waȟs Na wěn ni'yuh'geh.

3) Neh gwa iis, O Sha da gwĕn ni'yuh, a ga da wăs das dah goh: neh de gạah săoh goh, neh kuh de wak'noh ă g'wah tah. 
4 De wa gah săh twah'oh ha yah̄'da deh na Na yah'da gwěn ni'yuh oh gyĕăh dvk neh gwă noh, ha gat ho da dọh no no da gă yat nono da găah dih.

5 Oh ga dyas hă o gi dah; sa gyeh kuh; neh săh ăh Na yah'da g'wên ni'yuh dea găh nya do.

6 Tăh̆ăh tha khes ha nik neh wus hă niwặh nyah̆ eehs hă shă no yoh gweh da ge ak, neh de yo gwa do gah do dyı̌ĕ de jo twa dasea'driěh.

7 Des dat, O Sha da g'wěn ni'yuh; da gyah da guh, O Sgwěn ni'yuh: neh gai onih iis she yăh do ga gwe'goh nok ni goă swa ăs hadi goh sa geh: de sa yah goh kuh na di noh jah neh tặhạh dea wă ni'yuh kaah.

8 Ja go yah da gwat hah ho wăh Na yah dagrwèn ni'yuh: neh sa dăh swi yưs hăh gaeh ha di yah'da deh neh so g'weh dah.

\section{O I'WAII DE WUS' Hב̆ SĂ (PSALM XXIII.)}

$\sqrt{\text { EH Na yah da gwěn ni'yuh ha gas deis doh }}$ e) tăh'ăh thah da wa ga dọă jos.

2 Neh huh ha gyas hă oh heó'weh no geoh ji yuhs: neh huh kuh hea kas heh dyee negi yus.

3 Sa yah da gwas kuh na gat twaih shăh; hak nă chi neh kuh heo weh o'a deh neh dyiěh wa yeis dah.g'wah neh no să noh dyui wal. 


\section{PSALM XXIII.}

4 Do găs, neh huh gih săh ha gee neh yoa gó soh heo weh o dăgo'nos dọ neh găa yat tăh'ăh ta ge sha nik ne waet găh; neh săh'ăh heh iis ăh nyiěh; neh ne crook* (sa jot gaah) kuh neh sa dah di shăh neh de wak ni go dagwah.

5 Neh huh găs oh sa dek swa gwah shu dạh he ge got neh huh dea di ga nee nok ni goăswa ăs: neh kuh o noh soh gas neh ak noh ăgeh; neh na ga wăh ga wis'dah de wa dawă toh.

6 Gai wa gwěn ni yoh' ne wi vu' kuh nada ni dăgs'hah neh ă wok no dạ dyiěh sek he ni wă ni shạ gee ă go hek da neh huh kuh ă gih dyo dak ho noh sot Na ya da gwěn ni'yu hă yui'wa dă dyiěh.

* The Crook was a long piece of wood like the Indian Javelin with one end bent round like a cane-handle. It was used for helping sheep out of bad holes, and for pulling down limbs of trees so the sheep might reach the leaves. So God cares for us.

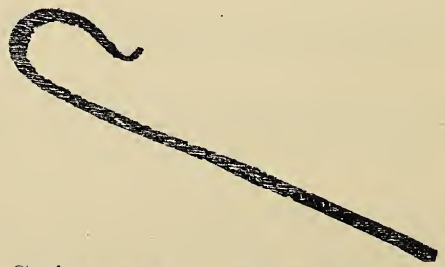

Sa jot gaa $\underline{a}$ (Shepherd's Crook.) 
O I'WAH DE WUS' HĂ GE'IH SGAEH. (PSALM XXIV.)

$N$ EH heh yoă'ja deh neh ho wăh Na yahoN da gwĕn ni'yuh, kuh heh ni yo gaah; heh yoă’ja deh, kuh heh ni yoo ya go heh.

2 Neh sặh'ăh kuh ho gặh săh goh heh ganyo dae nyoh, neh huh kuh ho ya daăh hehdoh no no dos hăh geh shoh.

3 Soo nah ot ă ye no dok dăh heóweh ni o wăh Na yah da gwĕn ni'yuh ? neh gih shăh neh so nah ot ă ye dak shoo no nok da do gặh dih geh?

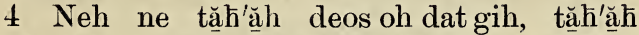
kuh deh oi wa eh na wĕn nyah sah geh: tăhh'ăh kuh deh neh huh da deoh gwah doh no twaishăh heo'weh ta wa des, dao no gwa nyok gihsăh gah ni go hoh das hă gọ.

5 Neh naeh ăo yah do went na dăh swi yos ăh ă dwah dă dih ha yah da deh Na yah da gwěn ni'yuh, neh kuh neh dye i wa yeis dah gwah ă dwoh dă dih ha yah dadeh Na wĕn ni'yuh neh sho yah da gwat thah.

6 Neh ne găh ga wa ji ya deh no wa yah di sas, na o sa goh sa găh, O Ja cob.

7 Swat ges gwa o $\mathrm{O}$ iis hoh ă gaă doh: neh kuh de swa deh gwah dook, iis neh wa oi'wà da dyiěh yeh ga ho gaă doh: neh dih ne Go wạgo wa heh de tgaă sa oh ă ta yoh.

8 Soo dih ne gạah nă gă Go wa go wa heh de tgaă sa oh? neh Na yah da gwěn ni'yuh ha deh jia tah hah has de sho wa năhi, neh $\cdot \mathrm{Na}$ - 


\section{PSALM XXIV. PSALM XXV.}

yah da gwěn ni'yuh hah has de sho wa năh neh a di yos hăh geh.

9 Swat ges gwao, $O$ iis hoh ăo gaă doh: haeh gwah gwa kuh de swah gwaht, iis neh wah oi wa da dyiěh geh ga ho gaă doh: neh ni deh Go wa yo wa heh de tgaạ sa oh ạ ta yoh

10 Soo dih ne găh nă gă Go wạ go wa heh de tgaă să oh ? neh Na yah da gwěn ni'yuh neh gă dyoh go wa năh, neh ne huh Go wa go wa heh de tgaă saoh.

\section{O I'WA\# DE WUS' HĂ WIS SGAEH. ( PSALM XXV.)}

IS, heh syah da deh, O Syah da g'wěn ni'yuh, de wa geh gwah dọ na gat wai săh.

2 O Swĕn ni'yuћ, gyah daahs heh syah da deh: sụ noh dih ă gah deăh, să noh nok nigoă swaăs het găh a yo gat hăh gwăh.

3 Do găas', să noh no nă nọ geăh doh heh syah da deh ă no deăh; neeh hă nódeăh neh do sa wă not gah was neh deh tah gwis dăh deht gie o nih.

4 Da gọ wat has; heh, nih syă noh dăăa $\quad 0$ Syah da g'wén ni'yuh; da gio nyăh heh sat hade nyoh.

5 Dak nă chii neh huh, hăo weh niis sawăh neh gai wa gwěn ni'yuh, neh kuh da gio nyăh; iis săh̆ăh Swěn ni'yuh neh swa gyahda gwat tah' neh huh dih a gă noh gea dô̆, heh syah da deh he ni wă ni sees. 


\section{PSALM XXV.}

6 Sa sas hăăt, O Syah da g'wěn ni'yuh, heh ni sa da ni dă o, neh kuh, ne sa no o gwah shăh he ni yo da ni dăo: heh neh săȟăh dayoi wa da dyiěh ne wa ga yoh.

7 Să noh, neh, o se'saa hăăt na gi wa nehakshăh̆ neh chi go gweh da seeh, do gaht neeh ne de go ya nes hăi da no nii: o da gasheh dak he ni sa da ni dăo da gas hăăh seek, o da gai o nih he ni wi yo heh syah da deh, O Syah da gwěn ni'yuh.

8 Wi yu neh kuh ne tgai wa yeih, Na yahda gwĕn ni'yuh: neh dih gai o nih ạ o goi o nă no di wa ne a goh hạa a neeh.

9 Neh goh ni goă ni dăs ăo go yah da do gặs heo weh de wa dyah do weh dah gwah; neh kuh ne goh ni goă ni dặs ăo gơ'i o nyăh he éh dah gwah.

10. Ga give goh heh nio taá deh Na yah dag’wèn ni yuh o da ni dăo gai wa gwěn ni'yuh kuh heo weh ni ye yah da deh ne goh să nonii no i wih sah hoh kuh no i wa gwai so gwăh.

11 Neh dih ne sah să noh o da yoi wah het O Syah da gwěn ni'yuh, dọ da gi wah să gwas na gi wa neh ak shăh; neh săh̆ ăh ne we soh.

12 Gaă nia yah da hos ha nis Na yah dagwěn ni'yuh? neh neh huh ăo i o nyăh heoweh gwa ạă goh.

13 Neh né huh naeh kuh notwaishăh ă yoh ni goi yoh hă sga no geek; neh kuh naa wa ji yăh ắo no wăs thehtt he yo ă ja deh. 


\section{PSALM XXV.}

14 Neh noh dah seh dôh geh no o wăh Nayah da gwěn ni'yuh ii neȟs no sha nis; neh kuh o yạ deet ăa go yo nyăh heh ni oi wih sah hoh.

15 Dyut gont niih dee ga neeh Na yah dagwěn ni yuh; heh neh sặh́ăh ăh sha gah sihda wih sih heoweh ni gah ă ot.

16 Do da sa dỉh sa de nih do da sat gah dăh heh gyah da deh, neh kuh ne dọn da gi dă ; neh săh'ăh niih neh deh thah gwis dăh dewa ga doh ho ga nyăh kuh.

17 Neh heh noh de yo doa'oh g'wáh da nih na gă nyah sah geh o dú dỵăo dyiěh: O donda gyah da dâh goh heh noh de wa ga do hoga nyăh.

18 Sat ga toh he ni wa gyo ya gă kuh he ni yo nook deh: do da gi wah să gwas kuh ga gwe goh heh ni wa gyăh hih syoh.

19 Des he yah do wet nook ni go ăs wa ăs; neh săh'a ho nont gah deh: neh kuh heh ni oges wa ăs gah nya gas deh.

20 O seh să no nih na gat wa is hăh, donda gyah da goh kuh; să noh̆ ă sge ga yahs na gadeă ; neh sūhăh heh neh huh he wagyăh neh gyah daas heh syahdadeh.

21 Da se gọdok ne yo gwe goh neh tgaiwa yeih neeh dă wa găh nya dọk: iis săh̆ăh go yă noh gea dộh.

22 Sae jah da goh ne Is rael, O Swĕn ni'yu ga grwe goh heh noh do doă ôh gwah da nih. 


\section{O I'WA\# DE WUS'HĂ YE IH SGAEH. (PSALM XXVI.)}

20 ON DA GYAH DO WET, O Syah da gwěnni'yuh; heh net huh gat ha'i neh heh nidwa gat da des ah dō: neh kuh he yah dăăhs Na yah da gwěn ni'yuh; neh dih gai o nih thah da wak hih sgoh.

2 Da ge gah ă yo nyo, O Syah da gwĕn ni' yuh, neh kuh ne da gek dọ: sek do ha ge gah gweh no da nyoh hae gwah kuh na gèn'nyahsah keh.

3 Neh săh ăhh ne sa no oh gwah să̆ kuh he ni yo dă ni dắ’o neh o ăn doo heh de ge ga neeh; neh kuh neh huh no da wa geh no odyiěh niis sai wa gwěn ni yvs'do shă go shōh.

4 Tăhhăh kuh theh a ga dyăh do gă neh deh wa des theh no gweh, tặhặh kuh ta yag'we ne da dih ni goạh seeh.

5 A ges waăh oh heh ni gă dyoh gwa yặh neh waet gặh niă no dye hah: tặh'ạh kuh dogă ta ya gwa dyă no di wa ne a goh.

6 Ăk no a eh neh ges oh dah ges hoh heó weh ne deh oi waeh; o năh' o gwe nyo dă gatwa da seh ne sa wăh ne ye yah don twat ha, O Syah da gwěn ni'yuh:

7 Da o năh o gwe nyo ăt gio wa năt ă gye ă dvk no wă noh ne de yo dă no nyoh gwah, ă ga tyo wih kuh ga gwe'goh heh ni yoi wană gwah dohs ne sai oh das hăh shoh.

8 Syah da gwěn ni'yuh, kno oh gwah heh no da găo dyiê̌ heh sno geh heh sa noh sot, 


\section{PSALM XXVI. PSALM XXVII.}

neh kuh he ni yo nok da yăh heh ni ga no geh niis sa wăh ne syah da no ôh goh.

9 Să noh do gă ăh syo ek na gat wais hăh no di wa neh a goh, kuh he goh heh do gă no no det gwăh̆ so să soh nă no gweh:

10 Neh săh'ăh há dihs oh da de nyoh tăh'ăh deh ga do găs thăh, neh kuh na di yăos dohgwa ha dis oh dah ga no hoh ne gaet gặt hah.

11 Da neh dih sho niih ah, neh hụh ă gat hai neh ă dwa ga dat des ah do ok: don dagyah da goh, neh kuh ne da gi dă sek.

12 Neh niih ne gah sih dah neh huh oshă dah oh heo weh shah de gadeh: neh̆ huh ne găa dyoh gwa go niih deă săo nyoh Na yahda g'wěn ni'yuh.

\section{O I'WA\# DE WUS'HĀ JA DUK' SGAEH. (PSALM XXVII.)}

NEH Na yah da g'wěn ni'yuh de wa ga de hat heh dah goh; soo nah ot ăe sha nik? neh Na yah da gwěn ni'yuh neh gah has dehgoh he goh heh; soo dih nah ot ăe sha nih sek?

2 No năh no di wa neh a goh, neh huh heyok dah oh nok ni goă swa ăs kuh ne waet gă deo ga dă no da nii, ho di yo heh gyah da deh naadis aah ne geh wá ah, dao nồ sih dyak ho di yah dỵe năh oh kuh.

3 Neh gih shăh ne găah dyoh go wa năh neh huh ăo di no da yạă iih don dáo ga do găh' do o dy̌ěh, neh na găn nyah sah tặh'ạh 


\section{PSALM XXVII.}

ta wăh ni go o nek: neh gih shăh na dih'yushăh dă wo ga do găh dăh niih, neh huh nă gă nôk gọ ga do găa sho heh năk ni goăh hos dek.

4 Joi wat de ya doă jo nis ôh Na yah dagwěn ni'yuh, neh dih ă gih sak seek; ne neh huh a gih dyo dak hò nó sot, Na yah da gwěnni'yuh, ga g'we'goh he ni wă nis hă ge ă goo hek na gat ga thuh heh ni yo yăs doh Na yah dagwên ni'yuh, neh kuh ăs ga dao do ok no nặ ogyeh ha tgă nis ah tah.

5 Neh să̆hăhh no năh dạ wo ga doă o hao'hwah ă o gyah dah set heo weh neh heh tgăhh tga deh : heo weh no dah seh doh geh hóh nigă os yoot, neh huh nō̆ goo hăo gyah dah set: neh huh ăo gih dyook gas dă

6 Da o năh nak noh ă geh dă gah gwah dook, heh tgăh hă ga dăh he nia di yah da deh nok ni go ă swa ăs de yo twa de see heh nigyah da deh : da o năh neh huh ăk dat no nigă os hă go ă gat gah he ni yo doh ho ok; ă ga dă no dăh, do găs', ă ga dă no dặh dặe ăh săonyo ok Na yah da gwèn ni'yuh.

7 Sat hon dek, O Syah da gwěn ni'yuh, gwă no năh dă gah să thoh ă gyeăh dok neh noh: da gi dă gwah huh, don da gwěn no să goh kuh.

8 No năh ăh sih, ji sak neh ge goh sah geh: neh na gă nyah sah ă să os, neh seh goh sahgeh, Syah da g'wěn ni'yuh, ă gih sak. 


\section{PSALM XXVII.}

9 Să noh we ăh nă dih sat goh sah seh dọ oh he ni gyah da deh; să noh neh huh ăe sis neh sah hah shăh neh ga noh kwăs hă goo: iis das gye no woh sea dyiểh; să noh ăs gyah do dih, să noh kuh ăs gat gah, O Swén ni'yuh na ga wăh neh ja go yah da gwat hah

10 No năh naah nih kuh ne noh yăh ăogat gah, da o năh Na yah da gwěn ni'yuh dă o gyah dak.

11 Da gio nyăh heo we gwa heeh seh dah gwah, O Syah da g'wĕn ni'yuh, neh huh kuh dak nă chi heo weh ni yoa do gat, neh dyoiwah nă gă nok ni goă swa ăs.

12 Să noh neh huh ăh sgah dat gah he nit huh dih ni go oh dạ nok ni goă swa ăs: neh săhhăh no nú wăh ooh wa di wa g’wai soh gwa ôh he gyah da deћ, neh kuh hă no doi chih tgăs no g'we nyo ă ya go nyoh.

13 A wak ni gọa ád dúh oh', a gă noh deh neh de a gi wa gwěn ni'yos dō̆ na gat ga thuh heni wi yoh Na yah da g’wĕn ni'yuh, taa ă ja deh neh yo heh o weh.

14 He să nô̆ geă do ok Na yah da gwèn niyuh: wi yoak heh nă sni go ăh has dek; neh kuh naeh ă yah has deh shonih ne să nyah sah geh: he să noh geă do ok, ga doh, Na yahda g'wěn ni'yuh. 


\section{O I'WAH DE WUS'HAِ DE GYIUH' SGAEH}

\section{(PSALM XXVIII.)}

N EH HUH hah dă gah să toh heh syah dadeh, O Syah da gwèn ni'yuh na ges dạ ăh : să noh tă sa dye ok heh gyah da deh: neh să ${ }^{\prime}{ }^{\prime}$ ăh ne dyăa gwah tă sa dyiěh gih săh heh gyah da deh̆, shah dă ya wăh he ni yoh dă ne wao no dyah dăh doo dyiěh heh o ya deh.

2 Sat hon dek ne gwă noh na ga da nih dăs dọh, no năh neh huh hah dă gah să toh heh syah da deh, no năh dă gă nĭs ôh dah gwat 'he gwa dyo nok da do găh dih.

3 , Să noh neh huh ăs gha wih do gă no diwá neh a goh, kuh no dioh deh gọ ne waetgăhh, neh sgă noh thi sa go di wặ nạ a sa năă nă noh sa ne gă noh, neh waeh ne waet găh nonă nyah sa gọ.

4 Das he yoh ăt gas he dak heh ni o di yă no wăhoh, neh kuh ăt gas he dak hé ni gai wa ne ak'shoh dă hă no dă noh geă tah: das he yoh ă gas he dak he ni yoi yoh da shoh dạ na dis oh dah; don da she yoh heh ni ga geohdă no not ga nyah gi eh.

5 Neh dyoi wah heh dă nos deis tah no ioh das hăh̆ Na yah da gwěn ni'yuh, kuh he ni yodo nyá noh nas oh dah, neh naeh ăo goi'wahdot, tăhh'ăh kuh tah do da go ges'guh.

9 Dea ăsa oh Na yah da gwěn ni'yuh, neh gai o nih heh ho too da dọh ne gwă noh ne chi wa ga da ni dăs dọ. 


\section{PSALM XXVIII. PSALM XXIX.}

7 Neh Na yah da g'wěn ni'yuh geh has degoh neh kuh a ga da wăh'das doh; neh na găn nyah sah ho yah dăă oh, a gye no woh se kuh: neh dih gai o nih na găn nyah sah̆ hahde yo dok ao es has; neh dih ă gye ăh dok na gă nọ ne deă săo nyo ok.

8 Neh Na yah da gwěn ni'yuh ha dih has degoh, neh kuh na o hwah ja go yah da gwas heh ni ah has de shôh dă no wa noh gaa.

9 Sas he yah da guh ne so gweh dah, sheya dăh swi yus dăh kuh he ni syah da g'wĕn ni yuh: she no dạ ok kuh dạs he yah dah gwah do ok hă yoi wa da dyiěh.

\section{O I'WA\# DE WUS'H SGAEみ屃.}

(PSALM XXIX.)

¿AS HE JOH Na yah da gwěn ni'yuh, O is neh swah să no wa năs, sas he joh Na yah da g'wĕn ni'yuh heh noh de aăh să êh kuh heh niah has de syoh dă.

2 Sas he joh Na yah da grwěn ni'yuh heh noh de a ăh să oh he ni yo wăh no sì noh; heji wa yă ni ak Na yah da gowén ni'yuh sa dyeah dah go ok he ni yo yăs doh no syo nya sạ dogăh dih.

3 Neh na wă nọ Na yah da gwěn ni'yuh neh huh ga eh no neh ga ges hoh̆; neh Nawĕn ni'yuh da săo gọoh ga wă no dahs: neh Na yah da g'wěn ni'yuh hea yah da deh ga g'we go he ni we suh' no ne ga nos. 


\section{PSALM XXIX.}

4 Neh na wă nọ Na yah da gwĕn ni'yuh gah has de sho wa năh; noh na wă noh Na yada gwěn ni'yuh ga no hoh neh gah has dehs hạ.

5 Neh na wă noh Na yah da g'wěn ni'yuh de ga yahs nos wah dah; do găs, neh Na yahda gwèn ni'yuh de a yahs nos wah dah neh tga no geh ne Leb a non.

6 Neh kuh neh huh nea yeh hah nă wa dwah tgat he ni ga ye hah not wais hă neh dyus gwa ont; neh kuh ne Leb a non kuh Sirion heh ni ga ye hah not wais hă neh dyusg'wa ont ot wais hă neh ga nyooh.

7 Neh na wă noh Na yah da gwěn ni'yuh de ga kah sos neh de wă ni wahs.

8 Neh na wă noh Na yah da gwěn ni'yuh ho daah thăh heo weh deh e no ge nyoh; neh Na yah da g'wěn ni'yuh ho daah thăh heh deh e no ge nyoh ne Ka desh.

9 Neh na wă noh Na yah da gwèn ni'yuh ni ga ye hah o năh ă wă no găt nă wa deh dooh neh dyus gwa ont yo dah sos kuh heh gah ha da yăh; kuh heo weh ni u noh sòt ga gwe goh ha dih snyet heh noh de aă săoh.

10 Neh Na yah da gwěn ni'yuh neh huh hăh dyoh no no dọ hăh geh; do găs', neh Nayah da gowen ni'yuh hặh dyoh go wăh go wa dyot gont;

11 Neh Na yah da gwěn ni'yuh ăo goh hosdes sho nih na oh gweh̆ dah shoh; neh Na yahda gwěn ni'yuh ă a go ya dah swi yos dạ na oh gweh doh sh̆oh a a a yeăh dvk neh sga no ti gai wi yuh'. 



\title{
A CHOICE COLLECTION
}

\author{
OF
}

ॐ ENGLISH HYVNSE

\author{
WITH TUNES.
}



Ised by permission of Wm. G. Fischer.

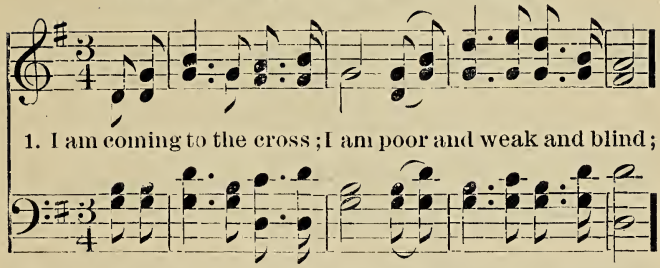

CH().-I am trustinu, Lort, in Thee, Dear Lamb of Calvary:

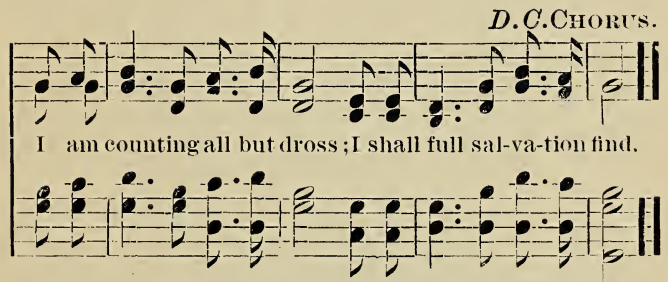

Humbly af Thy eross I bou, Sure me, Je-sus,save me uou.

2 Long my heart has sighed for Thee;

Long has evil reigned within;

Jesus sweetly speaks to me,-

"I will cleanse you from all sin."

I am trusting. Lord. ete.

3 In Thy promises I trust ;

Now 1 feel the blood applied;

I am prostrate in the dust;

I with Christ am crucifiel.

I am trusting. Lord, ete. 
Used by permission of John J. Hood.

(6)

1. Cast thy bread upon the wa - ters, le who have

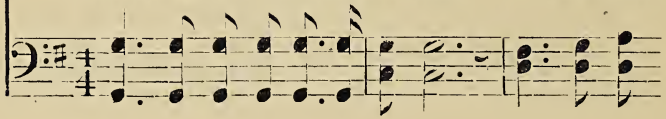

=1 -

but scant supply, An-gel eyes will watch above it;

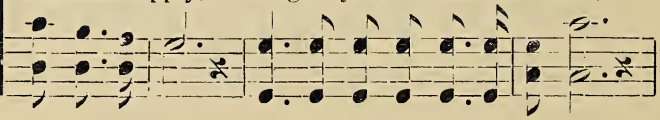

1.1

You shall find it by and by! He.who in his righteous

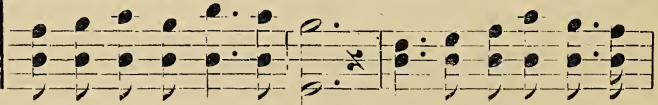

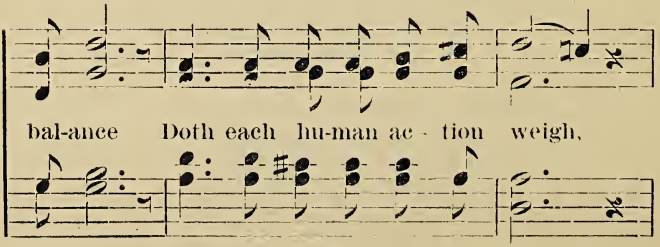



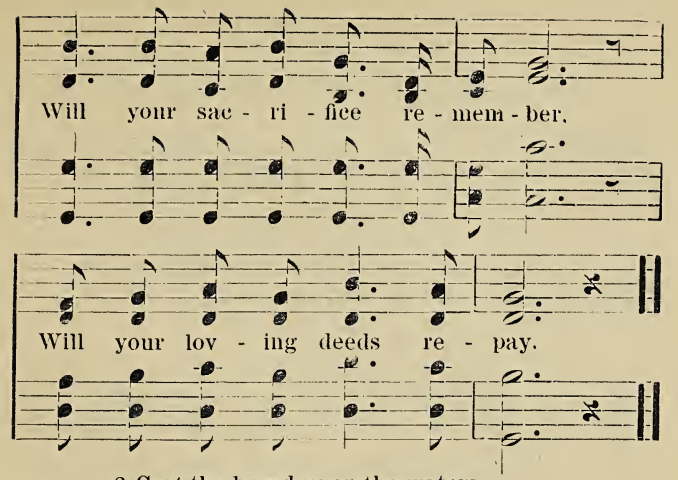

2 Cast thy bread upon tl.e waters,

Poor and weary, worn with care,-

Often sitting in the shadow.

Have you not a crimb to spare?

Can you not to those around you

Sing some little song of hope,

As you look with longing vision

'Thro' faith's mighty telescone?

3 Cast thy bread upon the waters,

Ye who have abundant store;

It may float on many a billow.

It may strand on many a shore;

You may think it lost forever.

But, as sure as fiol is true,

In this life or in the other,

It will yet return to you.

4 Cast thy bread upon the waters,

Far and wide yom treasures strew.

Scatter it with willing fingers,

Shout for joy to see it go!

For if you do closely keep it,

It will only drag you down;

If you love it more than Jesus.

it will keep' you from your crown.

5 Cast thy bread upon the waters.

Waft it on with praying breath.

In some distant, dombtful moment

It may save a soul from cleath;

When you sleep in solemn silence.

'Neath the morn and evening clew.

Stranger hands. which you have strengthened,

May strew lilies over you. 


\section{(OOI) BE WITH YOU.}

Used by permission of Rev. .J. E. Rankin, I). 1 .

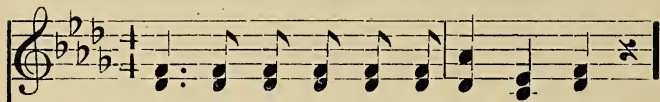

1. frod be with you till we meet a - gain,

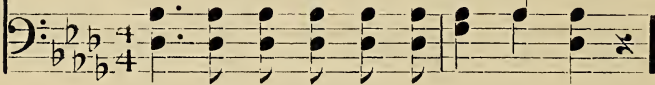

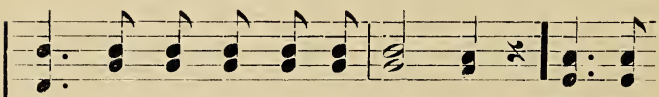

By His counsels, guide,up-hold you, With His

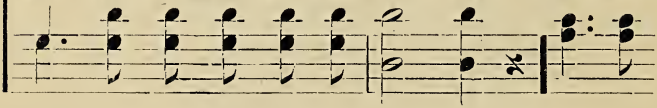

- 1 sheepse-cure-ly fold you, God bewith you till we

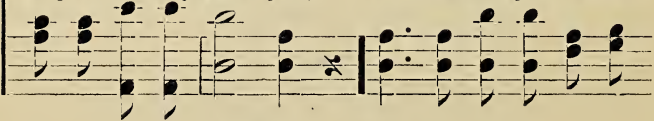

Cumrus. meet a-gain. Till we meet, . Till we meet.

T'ill we meet, till we meet a-gain,

FF B 


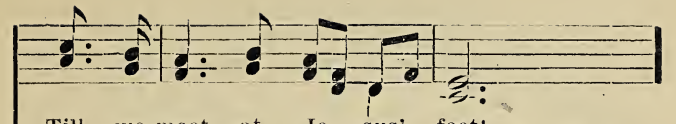

Till we meet at Je-sus' feet:

till we meet:
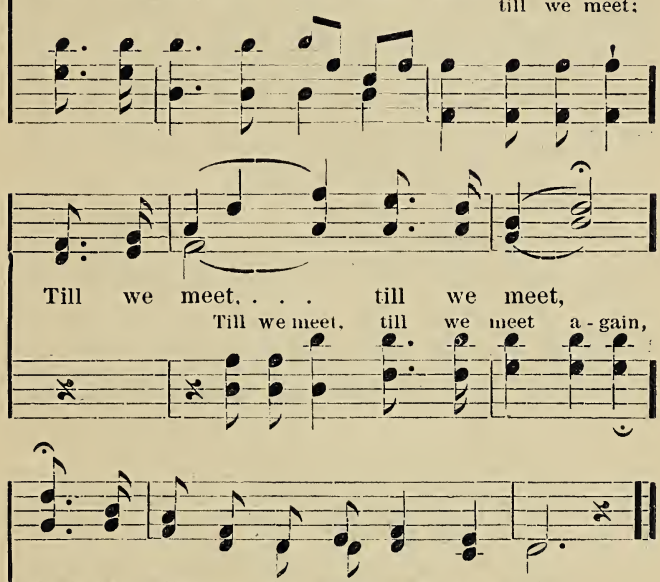

God be with you till we meet a - gain.

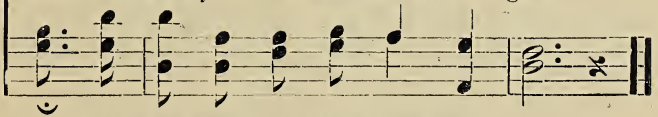

2 God be with you till we meet again,

Neath His wings securely hicle you;

Daily manua still divicle you;

God be witl you till we meet again.

3 God be witl you till we meet again.

When life's perils thick confonnd you;

Put $\mathrm{H}$ is arms unfailing round you,

God be with you till we meet again.

4 God be with you till we meet again,

Keep love's banmer flouting orer you;

Smite death's threatening wave hefore you, God be with you till we meet again.

$$
5 \text { Rev. I. E. RANKIN. }
$$




\section{BROKEN HEARTS.}

Used by permission of the Whyte Brothers.

J. M. W.

J. M. WHYTE.

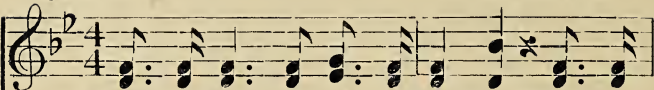

1. Like the mu - sic of a foun-tain, Which a

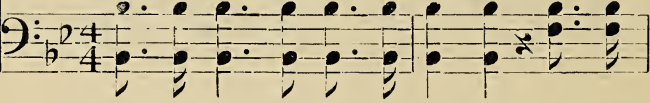

a.

thirst - y trav-'ler hears. Speaks a voice from Calv'ry's

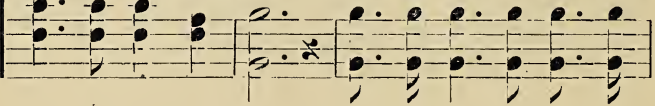

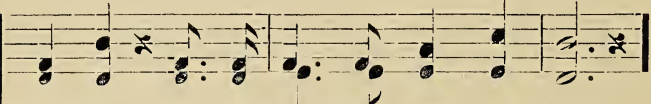

mountain, "I am morethan all thy fears."

\begin{aligned} 0 \\ -1 \\ \hline 1\end{aligned}

Cirorist.

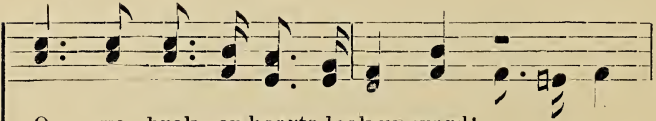

o ye brok-en hearts.look ul)-ward:

bro - ken hearts,

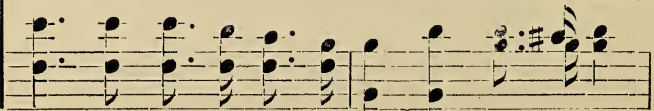




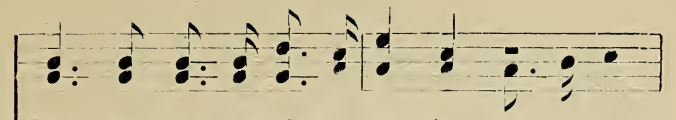

Hear the an-gel voic - es call- ing.
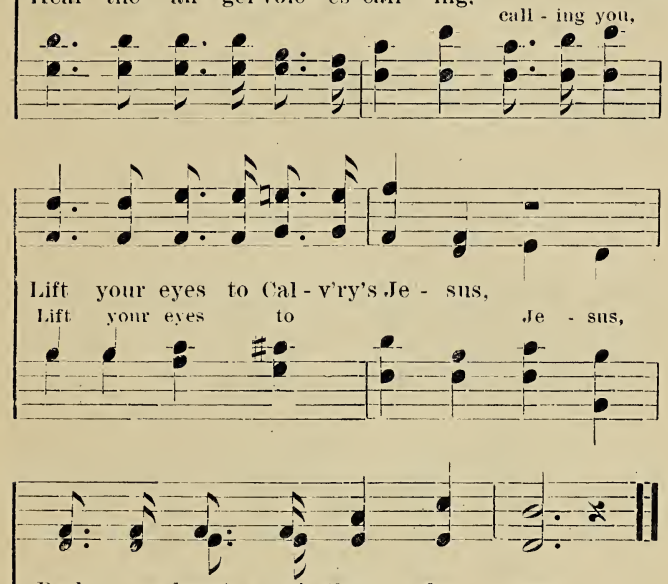

lirok - en - heart - ed there for you.

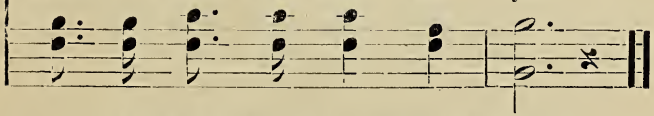

2 Though thy heart is crushed and broken, Like a storm-tossed ship at sea. Sinking, dying. Christ hath spoken. "It is $I$, look unto Me."

3 Though thy song hath naught but sorrow, Like a hiral's whose breast is torn;

Fly to ('hrist. nor wait the morrow. He liatli all thy sorrows borne.

4. Look away beyomd thy salness. Up to Jesus tulu thy gaze;

Then thy song shall turin to gladness Then thy tongue shall somind His praise. 


\section{ROOM FOR JESUS.}

"There uas no room for them in the inn." Luke ii: 7. Rev. J. W. SANBORN.

Rev. P. T. LYNN.

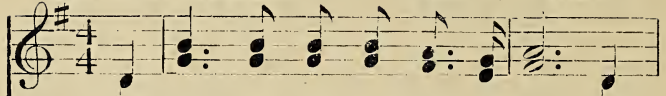

1. No room for Je - sus in the inn. The

$0=4$
4

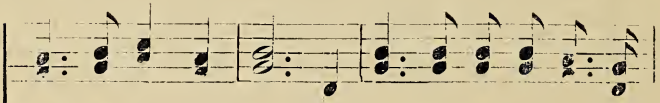

Son of God most high? And yet He bled to cleanse my 0
$R \rightarrow 0-1$

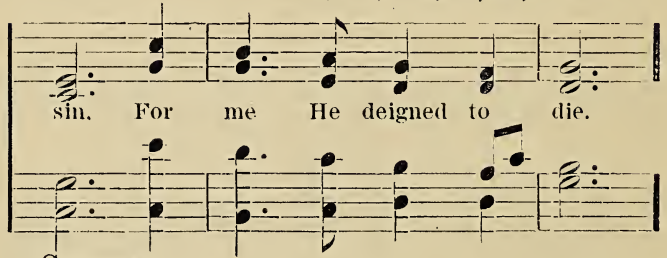

\section{Chorus.}

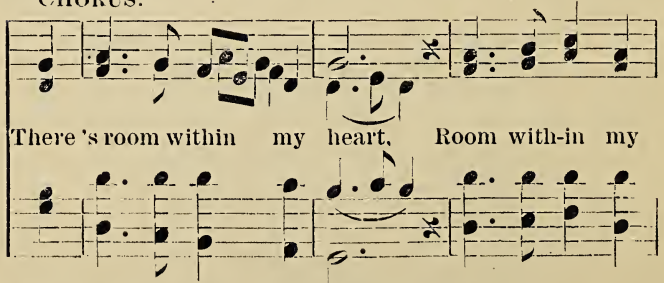

4th He's come into my heart. Coms a - to my lopyriahed, by sanborn and Lynn. 

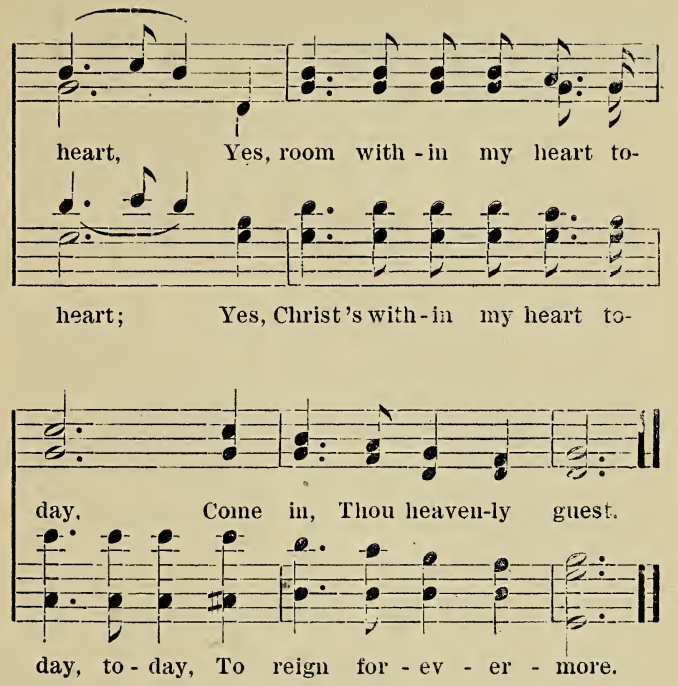

2 Shall I, like blinded Jews of old, Refuse an honored place.

To Him who left a throne of gold, To save me by His grace?

3 Thon Babe of Bethiehem. I pray. Come in, and dwell with me; Rule Thou my heart, to-day, to-day, lord, I would worship Thee.

+ I hear Him knocking at the door:

How sweet His loving plea:

Rejoice, my soul! the struggle 's o'er,

My Jesus feasts with me. 


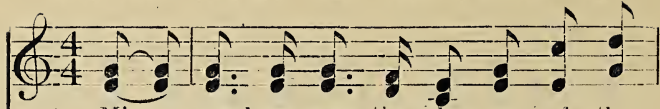

1. Mine eyes have seen the glo - ry of the

2. I have seen Him in the watch-fires of a

3. He has sommo-ed forth the trump)et that shall

4. In the be:tl-ty of the lil - ies Christ was

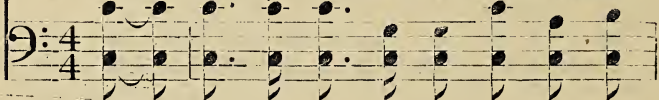

1-4.1-1-1

eom-ing of the Lork: IIe is tramp-ing out the hum-dred circ: - ling camms; They have buil'led Him an nev-er call re-treat; He is sift-ing out the boln a-cross the sea. ivith a glo-ry in His

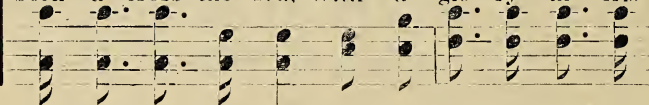
vin-tage where the grapes of wrathare stored : He hath al-tar in the ev-nimgdeivand (lamps; I have hearts of men bo-fore Ifis julgment seat: (oh. be bos-om that trans-fig-ures you and me; ls He

$\mid \begin{array}{ccc}-0 \\ y\end{array}$ -1. loosed the fateful lightning of His terrible guick sworcl: His read $\mathrm{His}$ righteons sentence by the dim and flaring lamps : $\mathrm{H}$ is swift.my soul.to an-s wer Him! be ju-bi-lant.my feet: ()ur died ti) make men ho-ly. let us die to make men free, While: 2010 2 


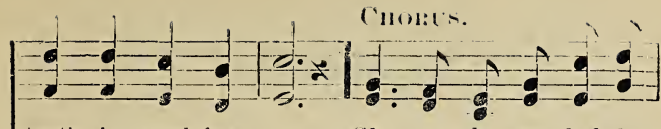
truth is march-ing on. (3lo- ry. glo-ry, hal-le day is march-ing on. fiost is marehting on. forl is morch-ing ont.

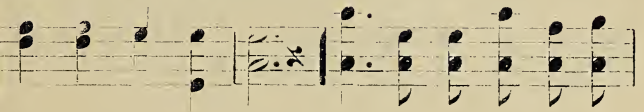

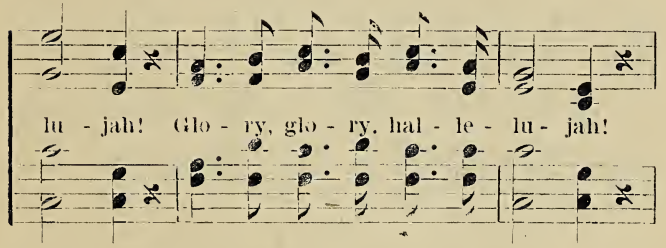
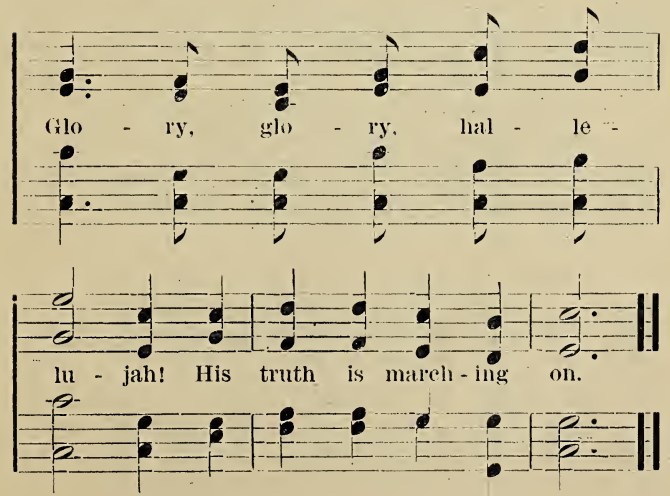
JESUS, THE LIGHT OF THE WORLD.

Used by permission of Geo. D. Elderkin. owner of Copyrighit.

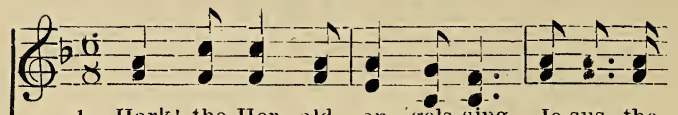

1. Hark! the Her ald an - gels sing, Je-sus, the

2. Joy - ful, all ye nid-tions, rise, Je-sus, the
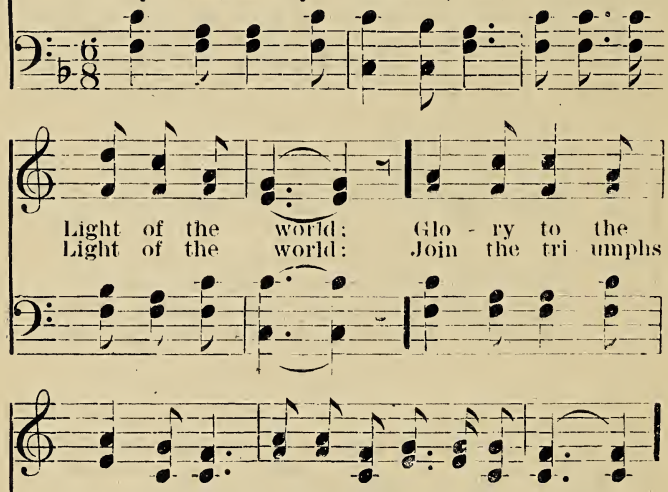

new-born King, Ie-sus, the Light of the world. of the skies, Je-sus, the light, of the world.

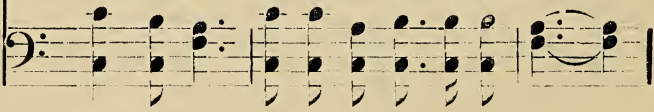

CiIt)initi.

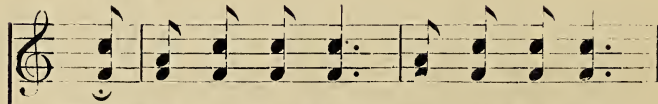

We'll walk in the light, beau - ti - ful Light,

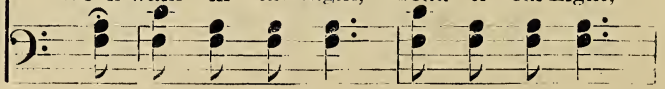




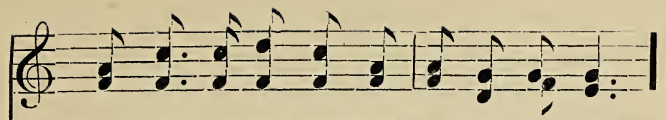

Come where the dew-drops of mer-cy are bright,
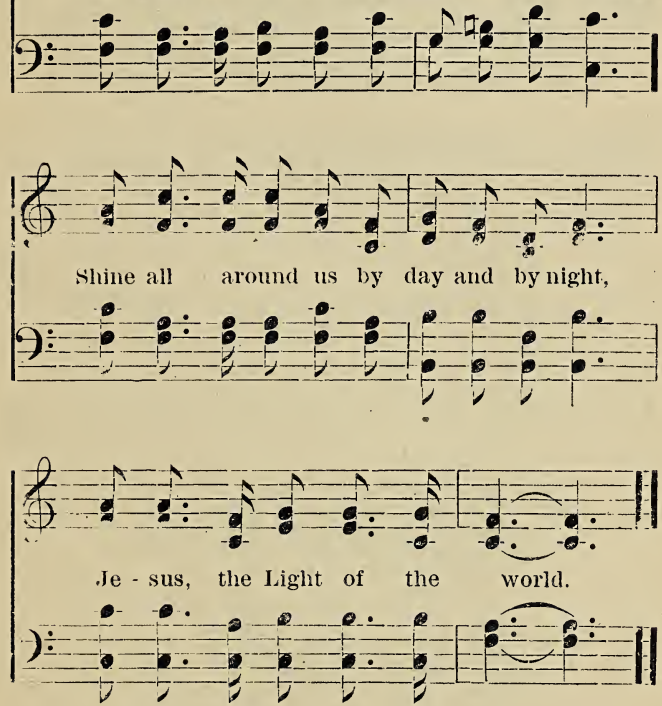

3 Christ by highest heaven adored, Jesus, the Light of the world;

Cirrist, the everlasting Lord, Jesus, the Light of the world.

4. Hail the heav'n-born Prince of peace, Jesus, the Light of the world ; Fiail the Sun of righteousness, Jesus, the Iight of the world. 


\section{EYE. ME.}

fised by permission of Riglow and Main.

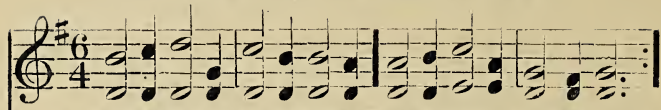

1. $\{$ Loril, l hear of showers of blessing Thou art seattering fill and fref: ?

1. Showel's, the thirsty land retreshing; Let some drops new fall on me, \}
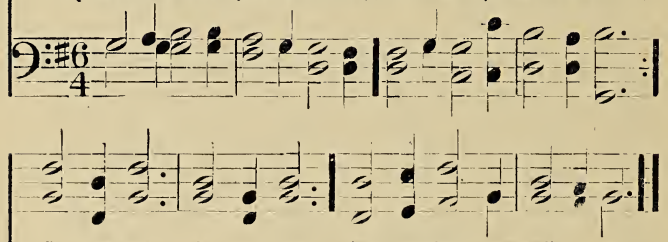

1. - vell me, l: - veal me, let some drops mis lall all me.

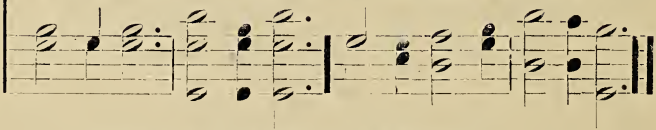

2 Pass me not. O (iod. my Father,

sinful though my heart mathe;

Thou mightst leave ne, but the rather

Let Thy inercy light on me.

Even me.

3 Pass me not, o gracious Saviour,

Let $m \rightarrow$ live and eling to Thee;

I am longing for "Thy favor:

Whilst Thon 't calling. oh, call me, Evell me.

4 Pass me not, 0 mighty spirit,

Thou canst make the hlind to see;

Witnesver of J'sus' merit.

Speak the word of power to me, Even me.

5 Love of (rod so pure and ehangeless. Blood of Christ. so rich and firee,

Grace of (iod. so strong and bommitess,

Magnify them all in me.

Even me.

MIS. ELIZAHETH CODNER.

Copyrizht. 1932, by Win. B. Brulbury . 
JUST AS I A.V, WITHOUT ONE PLEA.

Just as I am, without one plea, But that thy blood was shed for me, And that thou bidd'st me come to thee,

O Lamb of God, I come! I come!

2 Just as I am, and waiting not 'To rid my soul of one dark blot, To thee whose blood can cleanse each spot,

O Lamb of God, I come! I come!

3 Just as I am, though tossed about With many a conflict, many a doubt, Fightings within, and fears without, O Lamb of God. I come! I come !

4 Just as I am-poor, wretched, blind; Sight, riches, healing of the mind, Yea, all I need, in thee to find, O Lamb of lod, I come ! I come!

5 Just as I am-thou wilt receive. Wilt welcome, pardon, cleanse, relieve: Beanse thy promise I believe.

O Lamb of Gorl. I come ! I come!

6 .Just as I am-thy love minown Hath broken every barrier down; Now to be thine, yea, thine alone. O Lamb of God, I come ! I come! 
CTOSER, STILL CLOSER.

From "Crown of Life," by permission of W. W. Whitney.

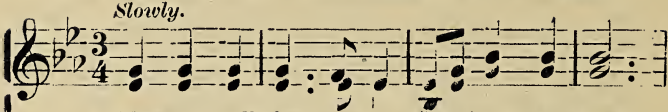

1. Clos-er, still clos - er. my Sav-iour, to thee,

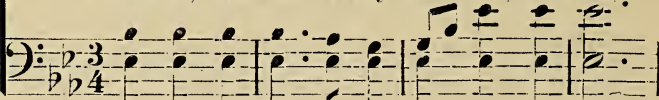

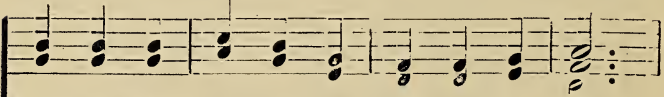

Clos-er to Je-sus fain, fain worlld I be;

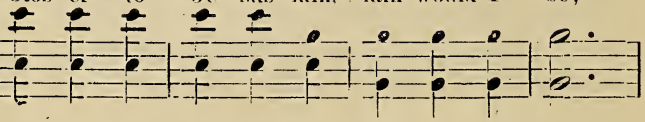

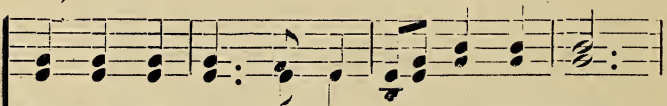

Round me his arm, on his bo . som my head.

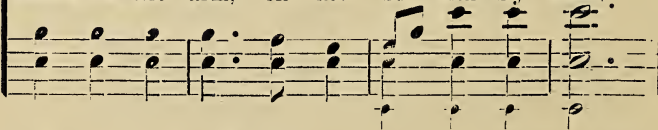

Eq

Near the diar side whichon Cal-va - ry bled.

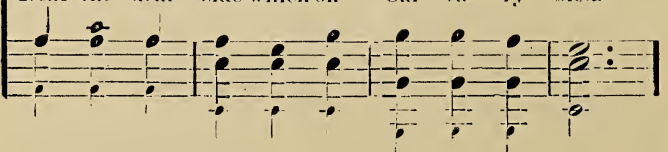


C'HORUs. cres.

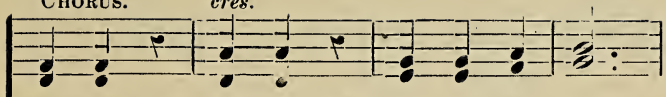

Clos-er, still clos-er, still clos -er to thee,
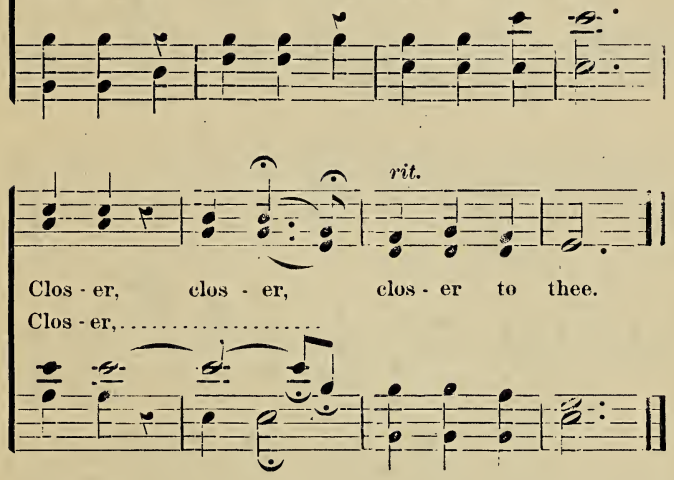

2.

Closer by day, though my sky be all bright, Closer, still closer when faileth th night; Earth has no spot where without him I'm safe, Time has no moment I need not his grace. Сно.-Closer, still closer, dic.

\section{3.}

When to the Jordan of death I descend, Danger I'll fear not, if Christ be my friend ; Breasting the billows, my death-song shall be, Closer, still closer, my Saviour to thee.

Cho._-Closer, still closer, \&c. 


$$
\text { IIOU FIRY A FOITDATIOX. }
$$

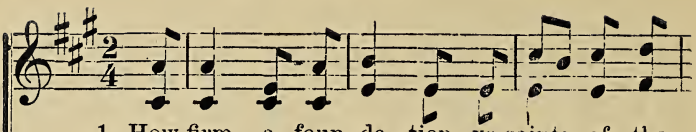

1. How firm a foun-da-tion, se saints of the

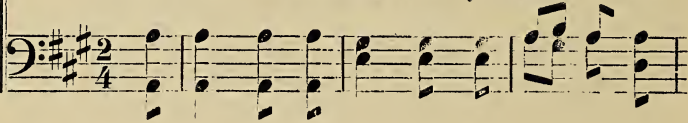

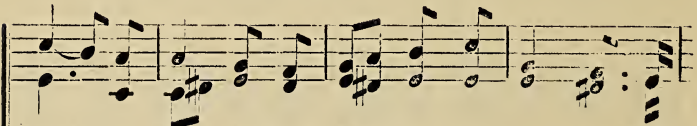

Lord, Is laid for your faith in lis ex - cel-lent
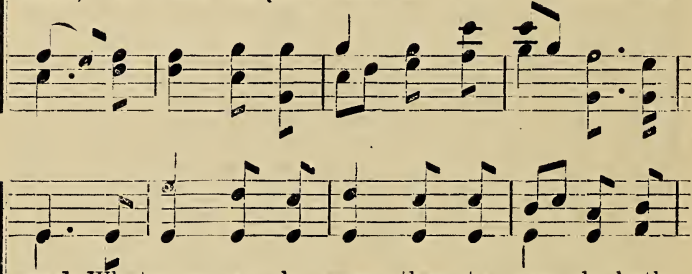

word: What more can he say than to you he hath
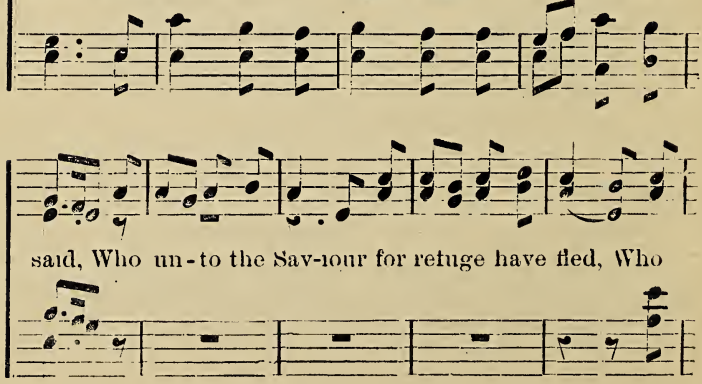


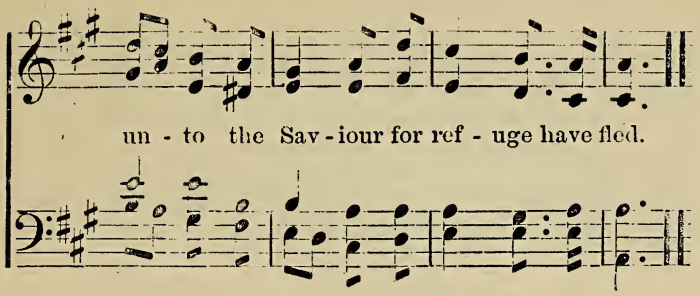

2. Fear not, I am with theo: Oh be not dismayed, For I am thy God, aurl will still give thee aicl: I'll strengthen thee, help thee, and cause thec to stand,

Uphell hy my righteous, omnipotent hand.

3. When throngh the deep waters I call thee to go, The rivers of sorrow shall not overflow; For I will be with thee thy trials to bless, And sanctify to thee thy deepest distress.

4. When through fiery trials thy pathway shall lie, My grace, all-sufficient, shall be thy supply: The flame shall not hurt thee; I only design Thy dross to consume, and thy gold to refine.

5. E'en down to old age all my people shall prove My sovereign, eternal, unchangeable love; And then when grey hairs shall their temples ador?, Like lambs they shall still in my bosom be borrc.

6. The soul that on Jesus hath leaned for repose, I will not, I will not desert to his foes: That soul, though all hell should endeavor to shake, I'll never, no never, no never forsake. Kirkham. 
THE MORNING LIGHT IS BREAKING.

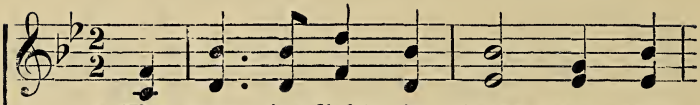

1. The morn - ing light is break - ing, The

2. Rich dews of grace come o'er us In
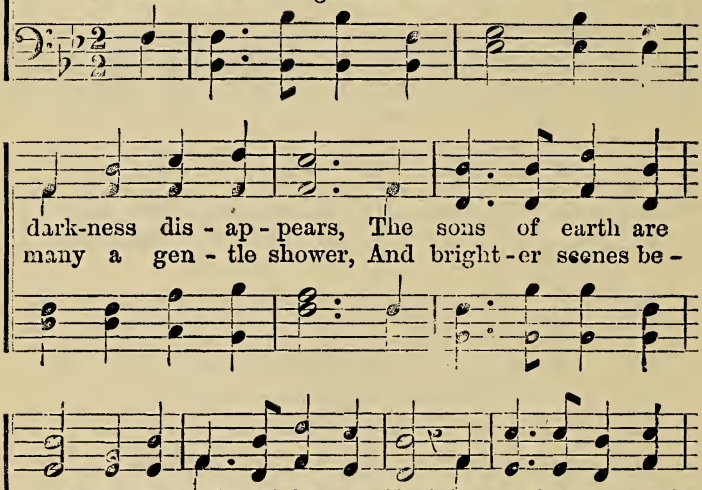

wak - ing To penitential tears : Lach breeze that sweeps the fore $\mathrm{u}_{3}$ Are opening eve-ry hour; Each cry to heav-en
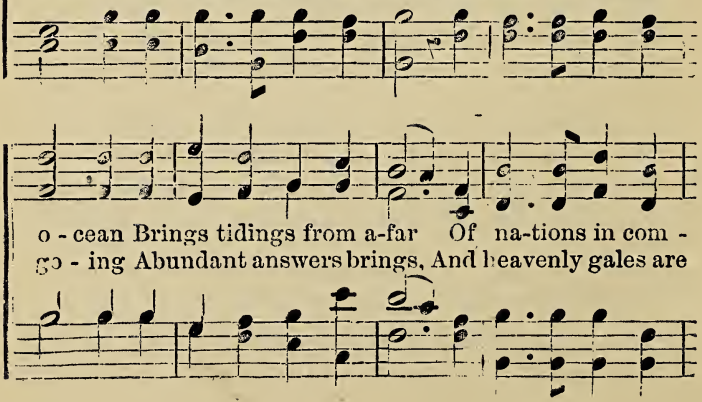


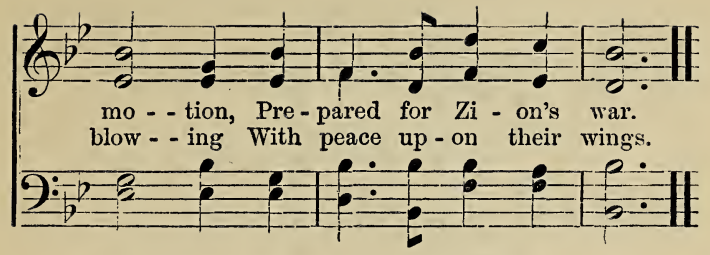

3. See heathen nations bending

Before the God we love, And thousand hearts ascending:

In gratitude above;

While sinners now confessing,

The gospel call obey,

A ad seek the Saviour's blessing-.

A nation in a day.

4. Blest river of salvation,

Pursue thy onward way, Flow thou to every nation,

Nor in thy richness stay;

Stay not, till all the lowly

Triumphant reach their home;

Stay not, till all the holy

Proclaim, "The Lord has come."

S. F. Smith.

NOW be the gospel banner

In every land unfurled;

And be the shout, Hosansa,

Reëchoed through the world:

Till every isle and nation,

Till every tribe and tongue,

Receive the great salvation,

And join the happy throng. Hastings. 
IIT DAYS ARE GLIDING SWIFTLY BY.

From SABEATH FIYMN AND TUNE BOOK, by permiski..:

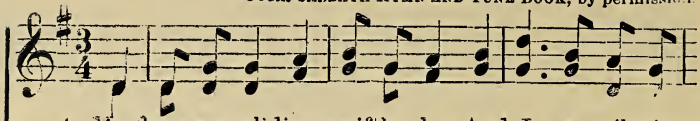

1. My days are gliding swiftly bv, And $\mathrm{I}$, a pigrim
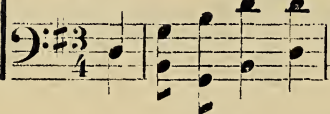

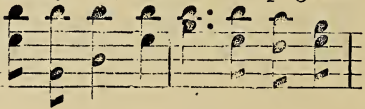

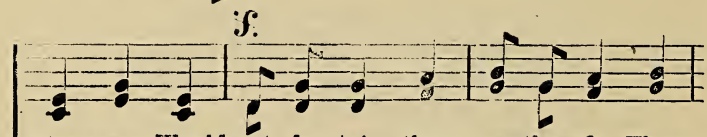

stran-ger, Would not de - tain them as they fly, Those

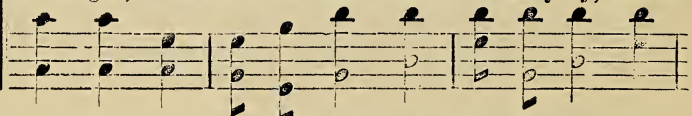

just be - forc, the shin-ing shore Ti:

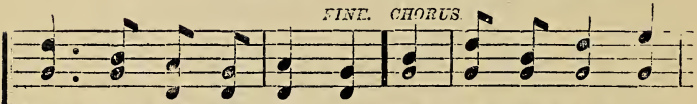

hours of toil and dan-ger: For Oh, we stand on

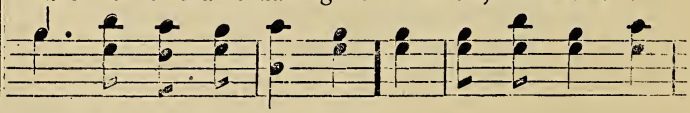

may al-most dis-cov - er.

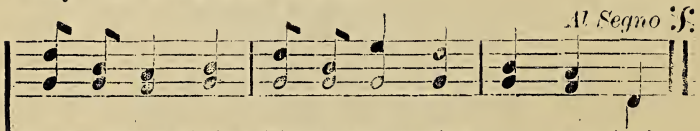

Jordau's strand, Ou fliend.; aro passing o - ver ; A...?

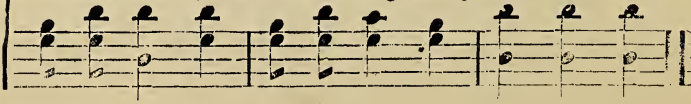


2 We'll gird our loins, my brethren dear, Our heavenly home discerning;

Our absent Lord has sent us word, Let every lamp be burning.-REF.

3 Should coming days be cold and dark, We need not cease our singing; 'That perfect rest naught can molest, Where golden harps are ringing.-REF.

4 Let sorrow's rudest tempest blow, Each cord on earth to sever; Our King says, "Come," and there's our home Forever, oh, forever.-REF.

David Nelson.

This piece, and the two which precede it are used by permission of the American Tract Society.

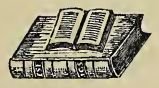

23 


$$
\text { COROXATION. C. M. }
$$

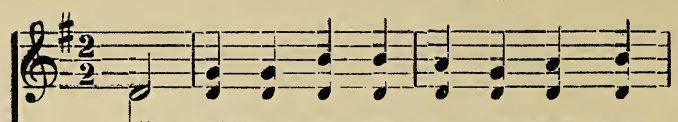

1. All hail the power of Je-sus' namp, Let
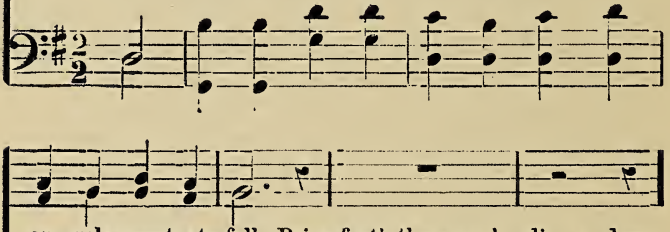

an-gels prostrate fall; Bring forth the roy-al di * a - dem,
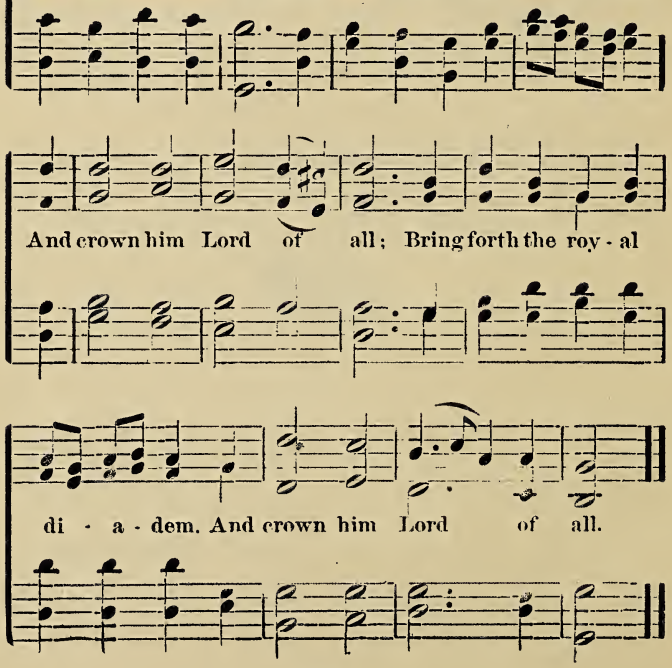
2 Crown him, ye morning stars of light, Who fixed this earthly ball;

Now hail the strength of Israel's might, And crown him Lord of all,

3 Ye chosen seed of Israel's race, Ye ransomed from the ifall,

Hail him who saves you by his grace, And crown him Lord of all.

4 Sinners, whose love can ne'er forget 'The wormwood and the gall;

Go, spread your trophies at his feet, And crown him Lord of all.

5 Let every kindred, every tribe, On this terrestrial ball, 'To him all majesty ascribe, And crown him Lord of all.

6 O that with yonder sacred throng We at his feet may fall!

We'll join the everlasting song, And crown him Lord of all.

Edward Perronet.

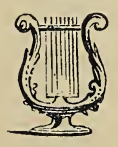





\section{INDEX OF FIRST LINES.}

HYMN。

To dă nọ'yăos Na wĕn ni'yuh

A gat'waih sa deh'sho nya'noo . . 59

A gěn'noh doh ho heh'o weh . 39

A gwáa wih a gyus'ho dah'gwah . 70

A naeh'ă swă eh̆, O swai'wa neh'a goh ? 25

A naeh' go waah' et hih ha dạăh 77

Cha găo'he dus ho'hah shăh shoh 65

Da gi'dă niȟ', neh Swěn ni'yuh . 31 .

Da go'want has, O Swěn ni'yuh 13

Dĭs dăht, O a gat'wais hăh 75

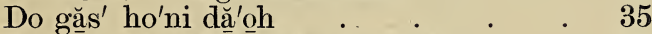

Do gă $\underline{a}^{\prime}$ skă noh̄ hă dwá na gek . 43

Do sa' swăhh ni goăn de nih 28

Dos găh' nih ah'dvk'his . . . $\quad 53$

Dyut gont' iih aeh' neh sek . . 50

Ga gwe'goh neh dwa na ${ }^{\prime}$ gé nyoh · 4

Ga gwe'goh neh dwa na 'age nyoh' Doxology。

Ga jih' iis sa da ni dắôh

Găn dyò gwah yặh o'ni dặh oh'

62

Ga oh'daa seh, ga oh'daa seh . . 22

Ga oh'da set', Sat wais'hi yu . . 19

Ga oh' da set', Se go wa năh . 3

Ga oh' da swet', do găă', wa doh 2

Ga oh' da'swet', iis, ne jo'gweh . 23

Gaoo'gwa' noo', ă ye'găh̆ . 26

Gih saas ne $\mathrm{da}^{\prime}$ yuh hat'he ak . . 41

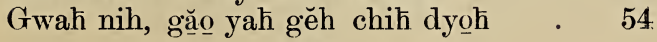


He'sga gont,' a gat'wa is hah

HYMN

Hes ga gont neh Sg'wa wěn ni'yuh

Ho di'ăh do'on dyiěh

Hot gwăh sai'oh Cho gyoh'hee dus : 15

Ho weh heh dyont gén'nis at'hah . $\quad 24$

Huk sah'dah shoh gowah go waa . . . 60

Tis nạah' de jo ăn'joh dăh' shoȟ . . 5

Iis neh snooh'gwah, Che yoh he dis . 55

Is, no'hah'shăh shō $\quad$. . . . . 7

Ja go yah da'gwat hah nogweh . . 20

Jat gat'huh he'ni sho'gwa wih . $\quad 34$

Je sus, Che yoh'he di's . , . 63

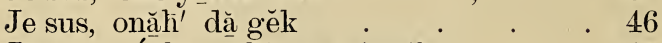

- Je sus sn'oh gwah'na gat'waih . . $\quad 47$

Kno ehs' nă ga dă nos'gwat hak 58

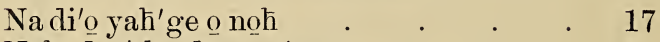

Neh des'she dwa no'nyo . . . . 51

Neh huh' năn dyiěȟ'gwa hó'weh . 72

Neh huh' ni yúo weh̆ he ga'wihs $\quad$. 12

Neh huh' no năh' oh gat'gat hòh 16

Neh na gah'shoh deh oh'dah was . . . 57

Ne sgáa hwah'soh neh he ni wehs . 71

Neh, waeh, neh Cha ga'o he dis . $\quad 30$

Ni yu'ne goh'dă no'ne gah̆ $\quad$. . . . 69

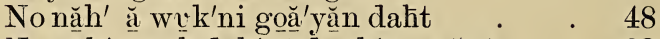

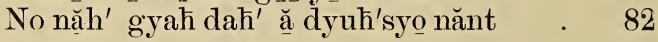

O agat'wa is'hăh . . . . . 14

O de dyá dặ no deeh' $\quad$. . . . . 37

Oh dyu'nih ek' dwah ni'goăh'gĕh $\quad 78$ 
HYMN

$\mathrm{O}^{\prime}$ gweh he ni ya'goh hoh'dă $\quad$. $\quad 67$

O gwe nyoog' găh', aga deăh'seek . . 44

Oȟ'ni goo'i yus deeh' no năh' $\quad$. . 40

Oi'wa neă gwat he'nah a'wăh 68

O Iis, ne Sai'wa yĕ'is doh . . . 81

O iis, Sat waih'chi yu, saăh'het . . 32

O năh' ho non'dăn da dyiěšs . 33

O năh' jo gwah'dă dyoon'dyiěh 84

O năh' tho hah ă skni go da goh $\quad$. $\quad 29$

O ni yoh' ga găh'săh'goh, swai'wi yus'doh 45

O să noh'do nyo hăs no gek . . $\quad 83$

Os'noo weh̆ o diăh doon dyiěh 76

O Swĕn ni'yuh, da g'wat'gat huh . 9

O Swěn ni'yuh, don da'gwat gah . $\quad 8$

O Swěn ni'yuh, Tis skno'oh gwah 38

$\mathrm{O}$ wăh nyah eh shoh teh gwă not . 1

Sa dáoh di yus, dyọă no gaah . 73

Sat'hon dek, a gat wais'hăh . 42

Se deh'ji ah ăh sat'hon dek . . 11

Sha yah'daat, neh no yah'do weh'dus hi yu 74

Si'gwa dyon da'deh sa doh gwah . $\quad 85$

Swa daoh'di yòs nee'ga gwe goh . 21

Swah'dăn dih̆, neh swai'o wa nănt 66

Swat'hon dek' dyiut haah' he o weh . 80

Swěn ni'yòh', neh no'ni dăă oh' $\quad$. 36

Syah da'gwèn ni'yuh da gwa yohss . . 52

Syăn ni yu, Syah̆'da gwěn ni yuh $\quad$. 49

Waă'eh ne Cha găo'hedus! . 18

Was ahs yoă jah ge chaa . . . . . 61 


\section{INDEX TO DOXOLOGIES.}

PAGE

Ga gwe'goh neh dwa na'ge nyoh' $\quad 77$ Wi yu' so nih' na gěn vah sah 77

O Is, nah Svaȟ'da gwě ni'yuh . 77

\section{INDEX TO THE PSALMS.}

PSALM I.

PAGE

PSALM II.

79

PSALM III. . $\quad . \quad$. $\quad$. $\quad$ 81-82

PSALM XXIII. . . . $\quad 82-83$

PSALM XXIV. . . . . $84-85$

PSALM XXV. . . . $\quad$. $85-87$

PSALM XXVI. $\quad . \quad$. $\quad$. $88-89$

PSALM XXVII. : . . . . 89-90

PSALM XXVIII. . . . . $92-93$

PSALM XXIX. . . . . . 93-94

\section{INDEX TO ENGLISH HYMNS.}

PAGE

"I am trusting, Lord, in thee." . 1

"Cast thy bread upon the waters." . 2-3

"God be with you till we meet again." 4-5

"Broken Hearts." . . . 6-7

"Room for Jesus." . . . 8-9

"Battle Hymn of the Republic." . 10-11

"Jesus, the Light of the world." . 12-13 


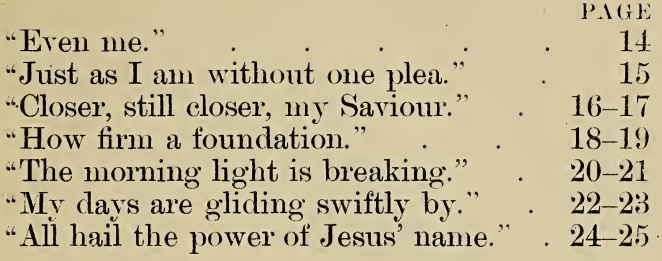




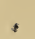

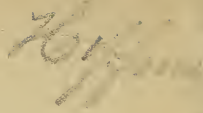





Microformed by

Preservation

\section{Services}

mim \#

June 1,1994

l 

